\title{
ALPRAZOLAM: profilo farmacoeconomico nei disturbi d'ansia e da attacchi di panico
}

\author{
Mario Eandi*
}

\begin{abstract}
Alprazolam is a triazolo analog of the 1,4 benzodiazepine class, widely used to treat patients with anxiety disorder, panic attacks and anxiety comorbidity with depressive disorder. Following oral administration, alprazolam is readily absorbed with peack plasma levels in 2 hours; extensive hepatic metabolism occurs and about $80 \%$ of the oral dose is excreted by kidney. The main metabolic route is hydroxylation catalyzed by cytochrome P450 3A: alpha-hydroxy alprazolam is partially active. Alprazolam possesses anxiolytic properties similar to other benzodiazepines; however, the triazolo ring confers to alprazolam a peculiar antidepressant and antipanic activity.

Anxiety disorder, panic attacks with or without agoraphobia, and mixed anxiety-depressive disorder represent an heavy economic burden to National Health System (NHS) and to society. In the present work the clinical pharmacology and the therapeutic profile of alprazolam are reviewed and analysed under the pharmacoeconomic perspectives of the italian patients, General Practitioners, NHS and society.
\end{abstract}

Farmeconomia e percorsi terapeutici 2002; 3 (1): 37-56

\section{INTRODUZIONE}

Alprazolam è un analogo triazolico della classe delle 1,4-benzodiazepine. Farmaco dotato di potente azione ansiolitica, è tra i più utilizzati in ambito clinico nel trattamento dei disturbi d'ansia, nei disturbi da attacchi di panico e nel disturbo misto d'ansia associata a depressione. $(1,2)$

In questo lavoro presentiamo un profilo farmacologico clinico aggiornato dell'alprazolam come base per alcune considerazioni di tipo farmacoeconomico.

\section{PROFILOFARMACOLOGICOCLINICO}

\section{Farmacocinetica clinica}

Alprazolam è una benzodiazepina utilizzata per via orale: viene prontamente assorbita dall'intestino inducendo concentrazioni plasmatiche di picco (Cmax) 1-2 ore dopo la somministrazione. Circa l' $80 \%$ della dose orale è resa biodisponibile ed il pasto non interferisce con l'assorbimento. (2)
Alprazolam è legato per circa l' $80 \%$ alle proteine plasmatiche e raggiunge lentamente, in 23 giorni, l'equilibrio di distribuzione. (2)

Il volume di distribuzione è elevato $(1,1-1,2$ $\mathrm{L} / \mathrm{kg}$ ) e risulta aumentato nei soggetti anziani di sesso maschile e nei soggetti obesi: questo risultato è dovuto all'elevata lipofilia di alprazolam che tende ad accumularsi nel tessuto adiposo, più abbondante nei soggetti obesi e tendenzialmente anche negli anziani. Questo riscontro, tuttavia, non sembra avere conseguenze cliniche e non risulta correlato a differenze di efficacia sedativa. $(2,3)$

Alprazolam viene abbondantemente metabolizzato, soprattutto a livello epatico. La principale via metabolica è rappresentata dall' idrossilazione catalizzata dal citocromo $\mathrm{P} 450$ 3A4. Tra i vari metaboliti prodotti, l'alfa-idrossialprazolam presenta un' attività farmacologica analoga all'alprazolam ma ridotta approssimativamente del $50 \%$ come potenza.

Circa l' $80 \%$ dell' alprazolam viene escreto per via renale, prevalentemente sotto forma di metaboliti. Una quota pari al $7 \%$ circa viene eliminata per via fecale.(4)

La cinetica di alprazolam è lineare nel range di dosi singole compreso tra 0,5 e $3 \mathrm{mg}$. 
Nei soggetti adulti sani l'emivita media è stata stimata in 11,2 hrs. Il metabolita attivo presenta un'emivita di eliminazione di poco inferiore a quella di alprazolam. (3)

L'emivita di eliminazione risulta mediamente aumentata nei soggetti anziani, nei soggetti obesi e nei soggetti con gravi epatopatie, come la cirrosi.(5-7)

La clearance sistemica di alprazolam è stata stimata in $76 \mathrm{ml} / \mathrm{min}$, e la clearance renale in 371 $\mathrm{ml} / \mathrm{hr} .(4,5)$ La clearance sistemica del farmaco tende a diminuire nei soggetti anziani, soprattutto per la tendenziale riduzione della funzionalità renale correlata all'invecchiamento, e nei cirrotici. $(2,8)$

L'azione ansiolitica acuta di alprazolam è discretamente correlata alle sue concentrazioni plasmatiche. L'effetto ansiolitico si manifesta entro 1-1,5 ore dalla somministrazione ed il range terapeutico nel disturbo da attacchi di panico è fissato nelle concentrazioni plasmatiche comprese tra 20 e $40 \mathrm{ng} / \mathrm{ml} .(8,9)$

Nel caso della depressione l'effetto terapeutico dell'alprazolam non è correlato con le concentrazioni plasmatiche e si manifesta con una latenza di almeno una settimana dall'inizio della terapia.(10)

\section{Farmacodinamica}

Alprazolam è una triazolobenzodiazepina che ha le proprietà farmacologiche tipiche delle classiche benzodiazepine clordiazepossido e diazepam: attività ansiolitica, ipnoinducente, miorilassante ed anticonvulsivante. (11)

Come tutte le benzodiazepine, alprazolam agisce mediante un legame specifico con un proprio recettore situato sul complesso recettoriale $\mathrm{GABA}_{\mathrm{A}}$ : la presenza della benzodiazepina, inducendo un cambiamento di conformazione al complesso proteico, favorisce il legame del GABA al recettore $\mathrm{GABA}_{\mathrm{A}}$ incrementando quindi la corrente ionica del Cle la polarizzazione della membrana neuronale. A differenza dei barbiturici, le benzodiazepine non attivano direttamente il recettore $\mathrm{GABA}_{\mathrm{A}}$ ma necessitano del GABA per poter agire.(12)

Le triazolobenzodiazepine, ed alprazolam in particolare, si differenziano clinicamente dalle altre benzodiazepine per una spiccata azione sul tono dell'umore ed antipanico. $(2,10)$

L'anello triazolico, che caratterizza la struttura chimica di alprazolam, è ritenuto responsabile di tre specifici meccanismi d'azione, non presenti nelle classiche benzodiazepine, in base ai quali viene giustificata la peculiare azione antipanico e sul tono dell'umore di alprazolam. $(13,14)$.

Alprazolam inibisce, con meccanismo competitivo, l'effetto di attivazione indotta dal
PAF sull'asse ipotalamo-ipofisi-surrene (HPA). L'azione anti-PAF è stata dimostrata in vitro sulle piastrine ed in vivo su modelli animali. Dopo somministrazione di alprazolam è stato osservato un diminuito rilascio di $\mathrm{CRH}$ dall' ipotalamo, con un aumento della sua concentrazione in quest' area ed una corrispondente diminuzione nel locus coeruleus e conseguente riduzione dell' attività di questo centro cerebrale.

Alprazolam, a differenza delle classiche benzodiazepine, è in grado anche di attivare il recettore $\alpha 2$-adrenergico e quest'azione comporta la riduzione dell'attività del locus coeruleus.

Infine, alprazolam induce un incremento nel rilascio di serotonina con conseguente aumento dell'attività serotoninergica, particolarmente importante per giustificare sia l'azione antipanico sia il miglioramento del tono dell'umore peculiare di questa molecola.

Nella genesi degli attacchi di panico sono ritenuti importanti il sistema GABAergico, il sistema noradrenergico ed il sistema serotoninergico. Su questi tre sistemi alprazolam esercita un significativo effetto di modulazione che, a livello del locus coeruleus, comporta una depressione dell'attività neuronale ed una correlata riduzione o abolizione degli attacchi di panico. (14)

Inoltre, l'azione anti-PAF di alprazolam, inibendo la liberazione di CRH, contribuisce in modo sinergico a bloccare il meccanismo di attivazione del locus coeruleus responsabile degli attacchi di panico. (14)

L'effetto del tono dell' umore di alprazolam viene da alcuni autori correlata alla sua capacità di incrementare significativamente la latenza della fase REM del sonno. Questo effetto viene indotto anche dagli antidepressivi triciclici e dagli IMAO ma non dalle classiche benzodiazepine. $(2,15)$

\section{Interazioni farmacologiche}

I maggiori problemi derivanti dall'interazione con altri farmaci dipendono dalle caratteristiche metaboliche di alprazolam. La prima tappa metabolica di alprazolam consiste nell'idrossilazione catalizzata dal citocromo P450 3A4. Molti altri farmaci utilizzano questo medesimo isoenzima per il loro metabolismo ed alcuni di essi possono inibirne l'attività catalitica oppure indurne la sintesi. In particolare, i farmaci che inibiscono il CYP 3A4 comportano una significativa riduzione della clearance di alprazolam e quindi un iperaccumulo che può comportare la comparsa di reazioni avverse.

L'associazione di alprazolam con potenti 


\begin{tabular}{|c|c|c|c|}
\hline $\begin{array}{c}\text { Farmaco } \\
\text { Interagente }\end{array}$ & $\begin{array}{c}\text { Descrizione } \\
\text { e meccanismo }\end{array}$ & $\begin{array}{c}\text { Conseguenze } \\
\text { dell'interazione }\end{array}$ & Provvedimenti \\
\hline $\begin{array}{l}\text { Chetoconazolo } \\
\text { Fluconazolo } \\
\text { Itraconazolo }\end{array}$ & $\begin{array}{l}\text { Forte inibizione del CYP } \\
\text { 3A4. }\end{array}$ & $\begin{array}{l}\text { Riduzione della } \\
\text { clearance e aumento } \\
\text { della concentrazione } \\
\text { tessutale dell'alprazolam. }\end{array}$ & Evitare l'associazione \\
\hline $\begin{array}{l}\text { Cimetidina } \\
\text { Fluoxetina } \\
\text { Fluvoxamina } \\
\text { Sertralina } \\
\text { Nefazodone } \\
\text { Claritromicina } \\
\text { Eritromicina } \\
\text { Josamicina } \\
\text { Roxitromicina } \\
\text { Troleandomicina } \\
\text { Omeprazolo } \\
\text { Propossifene } \\
\text { Contraccettivi orali }\end{array}$ & $\begin{array}{l}\text { Inibizione variabile del } \\
\text { CYP 3A4 e di altri } \\
\text { eventuali enzimi. }\end{array}$ & $\begin{array}{l}\text { Possibile riduzione della } \\
\text { clearance e possibile } \\
\text { aumento della } \\
\text { concentrazione tessutale } \\
\text { dell'alprazolam. }\end{array}$ & $\begin{array}{l}\text { Monitorare le reazioni } \\
\text { avverse di alprazolam } \\
\text { ed eventualmente ridurre } \\
\text { le dosi. }\end{array}$ \\
\hline $\begin{array}{l}\text { Amprenavir } \\
\text { Delavirdina }\end{array}$ & $\begin{array}{l}\text { Possibile interazione a } \\
\text { livello del CYP } 3 \mathrm{~A} 4 \text {, } \\
\text { non documentato } \\
\text { direttamente. }\end{array}$ & $\begin{array}{l}\text { Possibile aumento della } \\
\text { concentrazione tessutale } \\
\text { di alprazolam. }\end{array}$ & $\begin{array}{l}\text { Cautela, monitorare il } \\
\text { paziente per le eventuali } \\
\text { reazioni avverse. }\end{array}$ \\
\hline Ritonavir & $\begin{array}{l}\text { Iniziale inibizione del } \\
\text { CYP 3A4, corretta da } \\
\text { una successiva induzione } \\
\text { enzimatica. }\end{array}$ & $\begin{array}{l}\text { Possibile aumento degli } \\
\text { effetti di alprazolam. }\end{array}$ & $\begin{array}{l}\text { Monitorare gli effetti } \\
\text { sedativi di alprazolam. }\end{array}$ \\
\hline Carbamazepina & $\begin{array}{l}\text { Aumento del } \\
\text { metabolismo di } \\
\text { alprazolam }\end{array}$ & $\begin{array}{l}\text { Ridotta efficacia di } \\
\text { alprazolam }\end{array}$ & $\begin{array}{l}\text { Monitorare l'efficacia di } \\
\text { alprazolam ed } \\
\text { eventualmente } \\
\text { aumentare la dose. }\end{array}$ \\
\hline Propranololo & $\begin{array}{l}\text { Lieve aumento della } \\
\text { clearance di alprazolam }\end{array}$ & $\begin{array}{l}\text { Possibile ma non } \\
\text { documentata riduzione } \\
\text { dell'efficacia di } \\
\text { alprazolam. }\end{array}$ & $\begin{array}{l}\text { Monitorare l'efficacia di } \\
\text { alprazolam ed } \\
\text { eventualmente } \\
\text { aumentare la dose. }\end{array}$ \\
\hline Digossina & $\begin{array}{l}\text { Aumento documentato } \\
\text { delle concentrazioni di } \\
\text { digossina. }\end{array}$ & Tossicità da digossina. & $\begin{array}{l}\text { Monitorare i livelli di } \\
\text { digossinemia e ridurre le } \\
\text { dosi di digossina. }\end{array}$ \\
\hline Alcool & $\begin{array}{l}\text { Potenziamento degli } \\
\text { effetti depressivi e } \\
\text { riduzione della clearance } \\
\text { (effetto acuto) }\end{array}$ & $\begin{array}{l}\text { Aumento degli effetti } \\
\text { sedativi }\end{array}$ & $\begin{array}{l}\text { Evitare l'uso di bevande } \\
\text { alcooliche. }\end{array}$ \\
\hline $\begin{array}{l}\text { Danshen (Salvia } \\
\text { miltiorrhiza) } \\
\text { Kava } \\
\text { Magnolia (estratto) } \\
\text { Passiflora (estratto) }\end{array}$ & $\begin{array}{l}\text { Potenziamento degli } \\
\text { effetti depressivi sul } \\
\text { SNC. }\end{array}$ & $\begin{array}{l}\text { Aumentato rischio di } \\
\text { depressione del SNC. }\end{array}$ & Evitare l'associazione. \\
\hline $\begin{array}{l}\text { Caffeina } \\
\text { Teofillina }\end{array}$ & $\begin{array}{l}\text { Antagonismo degli effetti } \\
\text { di alprazolam }\end{array}$ & $\begin{array}{l}\text { Ridotta efficacia } \\
\text { sedativa ed ansiolitica di } \\
\text { alprazolam }\end{array}$ & $\begin{array}{l}\text { Monitorare l'efficacia di } \\
\text { alprazolam ed } \\
\text { eventualmente } \\
\text { aumentare le dosi. }\end{array}$ \\
\hline Iperico & $\begin{array}{l}\text { Antagonismo dell'effetto } \\
\text { miorilassante di } \\
\text { alprazolam. } \\
\text { Inibizione del CYP 3A4 }\end{array}$ & $\begin{array}{l}\text { Ridotta efficacia } \\
\text { miorilassante di } \\
\text { alprazolam e possibile } \\
\text { aumento di reazioni } \\
\text { avverse }\end{array}$ & $\begin{array}{l}\text { Monitorare l'efficacia e } \\
\text { la tossicità di } \\
\text { alprazolam. }\end{array}$ \\
\hline Valeriana & $\begin{array}{l}\text { Interazione col recettore } \\
\mathrm{GABA}_{\mathrm{A}}\end{array}$ & $\begin{array}{l}\text { Possibile aumento o } \\
\text { riduzione dell'efficacia di } \\
\text { alprazolam }\end{array}$ & $\begin{array}{l}\text { Monitorare gli effetti } \\
\text { terapeutici e le reazioni } \\
\text { avverse di alprazolam. }\end{array}$ \\
\hline Alosetron & $\begin{array}{l}\text { Dimostrata assenza di } \\
\text { interazioni } \\
\text { farmacocinetiche }\end{array}$ & Nessuna & Può essere associato \\
\hline Warfarin & $\begin{array}{l}\text { Nessuna interazione } \\
\text { riportata }\end{array}$ & $\begin{array}{l}\text { Non documentato: } \\
\text { improbabile }\end{array}$ & Nessuno \\
\hline
\end{tabular}

Tabella 1

Interazioni tra alprazolam ed altri farmaci o piante medicinali documentate nella letteratura disponibile.(16) 
inibitori del CYP 3A4, come il chetoconazolo e l'itraconazolo è controindicata e dovrebbe essere evitata.

L'associazione con altri inibitori meno potenti del CYP 3A4, come la cimetidina, la fluvoxamina ed il nefazodone, non è controindicata in assoluto ma, quando attuata, deve prevedere la riduzione della posologia di alprazolam. Si deve ricordare che anche il succo di pompelmo può indurre una riduzione della clearance di alprazolam per inibizione del CYP 3A4.

Interazioni significative con alprazolam possono verificarsi con sostanze di uso voluttuario come le bevande alcooliche ed i prodotti contenenti caffeina o altre droghe caffeiche.

Inoltre, recentemente sono state documentate interazioni anche con diversi prodotti erboristici o estratti di piante medicinali (es. iperico, kava, danshen, magnolia, passiflora, valeriana) talvolta presenti in integratori dietetici di larga diffusione. La maggior parte degli estratti di queste piante medicinali contiene principi attivi capaci di modulare l'attività gabaergica centrale, potenziando l'effetto depressivo delle benzodiazepine.

Nella tabella 1 riassumiamo le evidenze accumulate sulle interazioni tra alprazolam ed altri farmaci o piante medicinali.

\section{PROFILOTERAPEUTICO}

I prodotti farmaceutici a base di alprazolam, attualmente registrati in Italia, possono essere usati, secondo scheda tecnica, nel trattamento dell'ansia, dell' ansia associata a depressione, del disturbo da attacchi di panico con o senza evitamento fobico. Inoltre, sono anche indicati per bloccare o attenuare gli attacchi di panico e le fobie nei pazienti affetti da agorafobia con attacchi di panico.

\section{Trattamento dell'ansia}

Un' ampia documentazione clinica ha dimostrato che alprazolam, nel range di dosi comprese tra $0,75 \mathrm{mg}$ e $3 \mathrm{mg} /$ die, somministrato in dose refratte, è efficace nel trattamento delle sindromi ansiose. (17-19)

La dose di 0,25-1mg somministrata per via orale tre volte al giorno è risultata significativamente superiore al placebo nel controllare la sintomatologia ansiosa di pazienti ambulatoriali affetti da nevrosi d'ansia di grado moderato o grave. Gli effetti collaterali erano inferiori al placebo e consistevano prevalentemente in sedazione e secchezza delle mucose. $(17,18)$

Alprazolam è circa 10 volte più potente del diazepam come ansiolitico: in uno studio con- trollato, 1,5-3 mg/die di alprazolam sono risultati efficaci almeno quanto $10-30 \mathrm{mg} / \mathrm{die}$ di diazepam.(19)

Alprazolam, alla dose di 0,75-3mg/die, è risultato significativamente superiore al placebo anche nel trattamento dell'ansia di grado moderato o grave del paziente schizofrenico.(20)

Infine, alprazolam ha dimostrato di essere efficace e ben tollerato anche nelle sindromi ansiose generalizzate con comorbidità organiche e nella sindrome dell' intestino irritabile.(21)

L'efficacia ansiolitica si manifesta in genere rapidamente e la sintomatologia risulta già significativamente migliorata dopo soli 4 giorni di trattamento.(22)

\section{Disturbi di panico}

Benzodiazepine ad elevata potenza, antidepressivi triciclici ed antidepressivi SSRI sono le tre classi di farmaci che oggi vengono raccomandate come trattamento di prima linea del disturbo da attacchi di panico. (23) Nessuna delle tre classi di farmaci ha dimostrato una superiorità di efficacia. (24) Tuttavia i pazienti che soffrono di disturbo di panico sono particolarmente sensibili alle reazioni avverse dei farmaci e tendono a reagire con aumento dei livelli d'ansia che a loro volta facilitano gli attacchi di panico.(25) Gli eventi avversi agli antidepressivi triciclici ed anche agli SSRI sono percepiti in modo particolarmente fastidioso e causano un'alta frequenza di abbandoni della terapia soprattutto nelle fasi iniziali. $(23,24)$ Le benzodiazepine sono meglio tollerate ed il loro effetto ansiolitico contribuisce ad evitare un'eccessiva risposta ansiogena in caso di reazione avversa.

Alprazolam è la sola benzodiazepina ufficialmente registrata in Italia per il trattamento del disturbo di panico non complicato o complicato da agorafobia o da comportamenti di evitamento.

Alprazolam è in grado di alleviare o abolire gli attacchi di panico quando viene usato a dosi giornaliere mediamente più elevate di quelle ansiolitiche, che possono arrivare fino ai $10 \mathrm{mg}$ / die.(26-28)

Un recente lavoro ha dimostrato che i soggetti sofferenti di attacchi di panico hanno una sensibilità differente e più elevata ad alprazolam rispetto a soggetti sani (29). In particolare, la somministrazione in acuto di alprazolam, sia nei soggetti sani sia nei pazienti produce effetti simili per intensità ed evoluzione temporale. Al contrario, relativamente a sedazione ed a riduzione delle attività cognitive, mentre nei soggetti con disturbo di panico produce un marcato miglioramento del tono dell'umore, nei soggetti sani induce astenia ed intenso affaticamento. 
Inoltre, alprazolam, sia nei soggetti sani sia nei pazienti con disturbo di panico, induce un significativo aumento dell'ampiezza relativa dell'onda beta (range di frequenze da 13 a 30 $\mathrm{Hz}$ dell'EEG), ma i pazienti con attacchi di panico dimostrano una maggiore sensibilità alla benzodiazepina. (29)

Questi risultati concordano con precedenti evidenze cliniche e rafforzano l'ipotesi secondo cui il disturbo di panico sarebbe anche correlato ad una maggiore affinità e/o sensibilità del recettore delle benzodiazepine. $(30,31)$

In uno studio multicentrico condotto in doppio-cieco su 256 pazienti, alprazolam alla dose di $1-10 \mathrm{mg} /$ die ha dimostrato di essere significativamente superiore al placebo nel controllare il disturbo da attacchi di panico anche nei soggetti che denunciavano agorafobia con attacchi di panico.(32) L'efficacia clinica era già evidente dopo 2 settimane di trattamento e dopo 4 settimane 1' $82 \%$ dei pazienti trattati con alprazolam erano migliorati ed il 50\% era senza attacchi di panico. Circa un terzo dei pazienti richiedeva dosi di $6 \mathrm{mg} / \mathrm{die}$ di alprazolam. L'efficacia di alprazolam si manifestava con un riduzione o un controllo completo dei sintomi fobici, degli attacchi di panico spontanei o situazionali, dell'ansia, dei comportamenti da evitamento e dell'inabilità secondaria.

Non esistono studi clinici comparativi tra alprazolam ed antidepressivi SSRI, mentre eistono alcuni studi di confronto tra alprazolam ed imipramina.

In alcuni trials comparativi diretti alprazolam ed imipramina risultarono equiefficaci e significativamente superiori al placebo nel controllare il disturbo da attacchi di panico al termine del periodo di trattamento acuto di 6-8 settimane, ma la benzodiazepina agiva più rapidamente e produceva risultati significativi già entro 2 settimane, mentre con l'imipramina la latenza dell'effetto era di 3-4 settimane.(26, 33, 34) Inoltre, l'uso di alprazolam comportava una minore percentuale di drop-out nella fase iniziale del trattamento (11\% versus $41 \%$ ). (26)

In un recente studio l'efficacia di alprazolam è risultata sostanzialmente equivalente a quella dell'imipramina nel trattamento acuto del disturbo di panico in un piccolo gruppo di soggetti anziani, con età > 55 anni. Le dosi efficaci nell' anziano erano circa la metà di quelle utilizzate nei soggetti adulti giovani. (35)

Alprazolam ha dimostrato di essere invece più efficace dell'imipramina e del placebo nel controllare i sintomi fobici e l'ansia anticipatoria in pazienti affetti da attacchi di panico.(36)

Alprazolam agisce più efficacemente e più rapidamente sui sintomi correlati all'attacco di panico, come la gravità degli attacchi spontanei ed il comportamento di evitamento, mentre l'imipramina agisce più globalmente e con maggiore ritardo.(37)

Nei pazienti con disturbo di panico che rispondono al trattamento farmacologico nelle prime 6-8 settimane è opportuno attuare una fase di mantenimento di ulteriori 6-10 mesi di trattamento per consolidare il risultato positivo, indurre una remissione completa della malattia ed evitare recidive. $(23,26) \mathrm{Si}$ considera remissione completa la scomparsa degli attacchi di panico accompagnata dalla diminuzione dei livelli d'ansia, delle fobie e dell'inabilità e dall'aumento dello stato di benessere generale. (23)

Il trattamento farmacologico può essere sospeso durante la fase di remissione del disturbo di panico, allorchè il paziente si senta pronto ed abbia una situazione stabile di vita. (38)

L'efficacia clinica e l'utilità della fase di mantenimento con alprazolam sono state studiate in alcune ricerche cliniche in confronto con imipramina. $(33,34)$

Un gruppo di 106 pazienti affetti da agorafobia con disturbo di panico, da disturbo di panico con moderata risposta di evitamento o da disturbo di panico non complicato, sono stati inclusi in una fase iniziale acuta di 8 settimane di trattamento, assegnati a caso al trattamento con alprazolam o con imipramina o con placebo.(33) I soggetti che durante la fase acuta hanno evidenziato un miglioramento dei sintomi hanno ricevuto un ulteriore trattamento cronico di mantenimento di 6 mesi. Un numero significativamente superiore di pazienti trattati con alprazolam, rispetto a quelli trattati con imipramina o con placebo, è rimasto in trattamento al termine della fase acuta di 8 settimane ed ha fatto registrare una riduzione degli attacchi di panico e del disturbo fobico, ed un risultato terapeutico complessivamente migliore nel follow-up a lungo termine nei soggetti che avevano completato un ciclo di 8 mesi di trattamento. Tutti i soggetti che avevano completato la terapia di mantenimento a lungo termine (27 nel gruppo alprazolam, 11 nel gruppo imipramina e 10 nel gruppo placebo) erano asintomatici al termine della fase di mantenimento. Alprazolam è risultato efficace e ben tollerato ad una dose media giornaliera di $5,7 \mathrm{mg}$. L'imipramina è stata usata alla dose media giornaliera di $175 \mathrm{mg}$ con buona efficacia sul disturbo di panico, ma i soggetti trattati con il triciclico hanno denunciato reazioni avverse più fastidiose ed un minor livello di accettabilità della terapia. Durante la fase di mantenimento i soggetti trattati sia con alprazolam sia con imipramina non hanno sviluppato tolleranza farmacologica e non hanno aumentato le dosi. (33) 
I soggetti che avevano completato la fase di mantenimento sono stati sottoposti ad un periodo di trattamento scalare di 4 settimane per arrivare alla sospensione definitiva del farmaco.(34)

Il trattamento scalare è stato attuato in cieco, riducendo ogni 3-4 giorni di $1 \mathrm{mg}$ la dose di alprazolam e di $25 \mathrm{mg}$ la dose di imipramina.

Quasi tutti i soggetti trattati con alprazolam hanno evidenziato qualche sintomo d'astinenza ed il $33 \%$ non è riusito a sospendere definitivamente il farmaco. I sintomi d'astinenza da benzodiazepina tendevano a scomparire entro 3 settimane dalla sospensione del farmaco. Una minore percentuale di soggetti trattati con imipramina o con placebo ha fatto registrare sintomi d'astinenza. La difficoltà a sospendere il trattamento con alprazolam non era correlabile alla dose di alprazolam ma alla gravità del disturbo di panico all'inizio del trattamento.

Una percentuale dei soggetti che riuscirono a sospendere il trattamento farmacologico, variabile dal $18 \%$ al $35 \%$ e non significativamente differente nei tre gruppi trattati con alprazolam, imipramina o placebo, è andata incontro a recidiva del disturbo di panico entro 3-5 settimane dalla sospensione.

Al follow-up di 1 anno, combinando la casistica del gruppo che aveva completato la fase di mantenimento con quella dei soggetti drop-outs, la percentuale di pazienti in remissione $\mathrm{o}$ in trattamento con farmaci antipanico non era significativamente differente nei tre gruppi di trattamento rispettivamente con alprazolam, impramina o placebo.

Complessivamente questa ricerca evidenzia la necessità di un prolungato mantenimento nel trattamento del disturbo di panico e sottolinea l'utilità di alprazolam nel favorire la continuazione di tale fase evitando abbandoni precoci ed una maggior frequenza di recidive. (34)

Le difficoltà collegate alla sospensione del trattamento farmacologico, soprattutto con alprazolam, sono state discusse da vari autori. Alcuni studi hanno cercato di documentare l'utilità di associare la psicoterapia cognitivocomportamentale alla terapia farmacologica soprattutto per ridurre le posologie di farmaci utilizzati, ridurre la durata del trattamento farmacologico e per facilitare la sospensione del trattamento senza aumentare il rischio di recidive.(39-41)

\section{Sindrome mista ansioso-depressiva}

Alprazolam, a differenza delle altre classiche benzodiazepine, presenta una significativa efficacia sul tono dell'umore dimostrata in alcuni studi clinici controllati versus placebo o versus triciclici. $(1,10,42,43)$

In uno studio controllato randomizzato di 6 settimane versus imipramina, la dose media di $2,6 \mathrm{mg} /$ die di alprazolam ha dimostrato di essere equivalente alla dose media di $128,4 \mathrm{mg} / \mathrm{die}$ di imipramina nel controllare i sintomi di pazienti affetti da depressione primaria. Non è tuttavia chiaro quale fosse il tipo di depressione dei pazienti inclusi nello studio. Il controllo dei sintomi era più rapido con alprazolam ed era già evidente dopo una settimana di trattamento. Il profilo di tollerabilità era migliore con alprazolam per la mancanza degli effetti anticolinergici tipici degli antidepressivi triciclici.(10)

In uno studio controllato versus imipramina e versus placebo, entrambi i farmaci hanno evidenziato efficacia clinica superiore al placebo. Alprazolam, tuttavia, è risultato più efficace nel controllare i sintomi somatici della depressione.(44)

In uno studio condotto su pazienti depressi ricoverati, alprazolam ha indotto un più rapido controllo iniziale soprattutto dei sintomi vegetativi senza ulteriori miglioramenti dopo 10 giorni di trattamento, mentre l'imipramina ha indotto un controllo più ritardato ma progressivo fino ad essere superiore sia per quanto riguardava i sintomi cognitivi che i sintomi vegetativi.(45)

In altri studi, pazienti affetti da disturbo depressivo maggiore hanno evidenziato una migliore risposta iniziale ad alprazolam, ma l'imipramina ha dimostrato di essere più efficace a lungo termine. Pazienti che oggi classifichiamo come depressi sottosoglia hanno risposto meglio all'alprazolam rispetto all'imipramina.(46)

Pazienti depressi con comorbidità del disturbo da attacchi di panico rispondono in modo equivalente sia ad alprazolam sia ad imipramina.(47)

Il confronto versus amitriptilina ha dimostrato che l'amitriptilina è più efficace nel trattamento della depressione maggiore nel sottogruppo di depressi in cui erano presenti sintomi di ansia, rallentamento psicomotorio, ma assenti fattori precipitanti, mentre l'efficacia era equivalente nel confronto generale.(48)

In altri studi non controllati l'efficacia sul tono dell'umore di alprazolam è stata confermata in varie forme di depressione. $(43,51,52)$

L'azione sul tono dell'umore di alprazolam è una peculiarità di questa molecola e non è presente in altre classiche benzodiazepine come il diazepam. $(19,53)$

Pertanto, alprazolam si afferma quale miglior candidato da associare ad un qualunque trattamento antidepressivo per il controllo della sintomatologia somatica nelle varie forme di depressione e nelle forme miste ansiosodepressive. 
Tollerabilità e sicurezza

Alprazolam è generalmente ben tollerato $\mathrm{a}$ dosi terapeutiche ed il suo profilo di sicurezza risulta eccellente.

Le reazioni avverse si manifestano per lo più all'inizio di un trattamento e regrediscono continuando la terapia. Gli effetti collaterali e le reazioni avverse ad alprazolam sono generalmente ben tollerate e vengono per lo più accettate dai pazienti che raramente sospendono il trattamento. $(54,55)$

Le reazioni avverse più frequenti consistono in un'accentuazione degli effetti farmacologici dell'alprazolam a livello del SNC e sono rappresentate da sonnolenza, depressione, torpore, stanchezza, vertigini. Talvolta il paziente manifesta fenomeni paradossi come cefalea, insonnia, irrequietezza. In rari casi sono stati evidenziati episodi di iperaggressività.

L'amnesia anterograda, comune a tutte le benzodiazepine, si manifesta soprattutto a dosi elevate e consiste in un disturbo nell'apprendimento di nuove informazioni, ma non coinvolge la memorizzazione di informazioni o di eventi accaduti prima del trattamento. L'amnesia può persistere per lungo tempo, ma tende a regredire completamente.

Gli effetti a lungo termine delle benzodiazepine sulle funzioni neuropsicologiche sono state oggetto di vari studi che consentono di escludere danni permanenti. Un recente studio controllato versus placebo, condotto su un gruppo di soggetti affetti da disturbi di panico, ha dimostrato che dosi di alprazolam di 4mg/die somministrate per 6 settimane non inducevano alcun deterioramento dei parametri di apprendimento, della memoria verbale, del tempo di reazione e di altri parametri neuropsicologici. (56)

Risultati simili sono stati ottenuti anche da altri gruppi di ricerca in soggetti affetti da agorafobia con attacchi di panico (57).

L'assenza di effetti a lungo termine di alprazolam sulla memoria è stato anche dimostrato da Kilic e collaboratori, che hanno seguito per 3,5 anni un gruppo di pazienti affetti da agorafobia ed attacchi di panico. (58)

La rassegna della letteratura indica che $\mathrm{i}$ pazienti trattati correttamente con una benzodiazepina raramente diventano consumatori abituali o dipendenti dall'ansiolitico loro prescritto.(59)

Il rischio e la gravità dell'eventuale dipendenza sembrano essere maggiori nei pazienti trattati con dosi di alprazolam superiore ai 4 $\mathrm{mg}$ /die per oltre 12 settimane. Sono stati descritti rari casi di farmacodipendenza da alprazolam, la maggior parte dei quali in soggetti affetti da disturbi di ansia associata a de- pressione e trattati con dosi di alprazolam tra 2 e $12 \mathrm{mg} /$ die per periodi prolungati dai 6 mesi ai 3 anni. In tutti questi soggetti alla sospensione del trattamento con alprazolam si è manifestata una sindrome d'astinenza.

La sindrome d'astinenza da alprazolam si verifica più frequentemente entro 24-72 ore dalla sospensione del trattamento e consiste in un corteo sintomatologico che può comprendere convulsioni, stato epilettico, ansia, attacchi di panico, sindrome paranoide, delirio, tachicardia ed ipertensione. $(60,61)$

\section{CONSIDERAZIONI FARMACOECONOMICHE}

Le benzodiazepine sono la classe di psicofarmaci maggiormente prescritti nei paesi industrializzati e rappresentano oltre il 50\% della spesa farmaceutica per farmaci psicotropi.(62)

Il campo di utilizzo clinico di alprazolam è molto vasto e riguarda tre principali disturbi psicopatologici di grande impatto sanitario e sociale, come il disturbo d' ansia, il disturbo da attacchi di panico e la sindrome mista ansiosodepressiva. Si tratta di disturbi che nella maggior parte dei casi vengono osservati e gestiti dal medico di Medicina Generale.

Un'importante ricerca epidemiologica condotta dall'Organizzazione Mondiale della Sanità (OMS) in 15 Paesi ha evidenziato che il 24\% dei pazienti afferenti agli ambulatori di medicina generale presenta un disturbo psichiatrico diagnosticabile secondo i criteri dell'ICD10 , un ulteriore $9 \%$ soffre di un disturbo sottosoglia, cioè di un disturbo in cui sono presenti sintomi psichici in almeno due aree diagnostiche senza che si raggiunga una soglia sintomatologica minima per una diagnosi formale; infine, il $31 \%$ dei soggetti presenta sintomi psichiatrici isolati, poco definibili in sindromi cliniche specifiche, ma nel complesso rilevanti sul piano della sofferenza soggettiva.(63)

Nel campione analizzato dallo studio dell'OMS sono state rilevate le seguenti prevalenze:

$\begin{array}{lr}\text { depressione } & 10,4 \% \\ \text { disturbo da ansia generalizzata } & 7,9 \% \\ \text { neurastenia } & 5,4 \% \\ \text { uso patologico di alcool } & 3,3 \% \\ \text { dipendenza da alcool } & 2,7 \% \\ \text { sindrome da somatizzazione } & 2,7 \% \\ \text { distimia } & 2,1 \% \\ \text { disturbo panico } & 1,1 \%\end{array}$

Il 9,5\% del campione soffriva di due o più disturbi psichiatrici (comorbilità). 
Nella figura 1 sono rappresentate le percentuali di prevalenza dei disturbi d'ansia, dei disturbi depressivi e dei disturbi misti rilevati dallo studio dell'OMS.(64)

La contemporanea presenza di ansia e depressione è stata confermata da vari autori, ed in alcune indagini raggiungeva il $13 \%$ dei pazienti afferenti agli ambulatori di medicina generale.(64-67)

Di particolare interesse risulta la sovrapposizione tra i due gruppi sintomatologici nelle forme sottosoglia, maggiormente presenti nella popolazione anziana, e nei soggetti affetti da patologie organiche: in questi casi la prevalenza del disturbo misto d'ansia e depressione risulta essere del $10 \%$ ed un ulteriore $8.1 \%$ presenta un'analoga sindrome sottosoglia. $(68,69)$

I dati epidemiologici dimostrano che molti sono $\mathrm{i}$ casi in cui l'ansia può sfociare in una forma mista di ansia e depressione: soggetti che durante l'infanzia o l'adolescenza hanno presentato un disturbo d'ansia presentano un rischio più elevato di sviluppare episodi depressivi nei 10 anni successivi. Inoltre, la comorbilità di ansia e depressione si manifesta con sintomi depressivi più accentuati ed è associata ad un aumento significativo del rischio di suicidio.

\section{Il costo di malattia del disturbo d'ansia}

Nonostante il disturbo d'ansia abbia una prevalenza molto elevata e superiore a quella di altre diffuse patologie psichiatriche come la depressione, il suo costo di malattia è stato raramente stimato. Un recente studio ha valutato il costo di malattia dei disturbi d'ansia negli Stati Uniti, basata sulla prevalenza della malattia, assumendo la prospettiva della società ed adottando la metodologia prevista dalla teoria

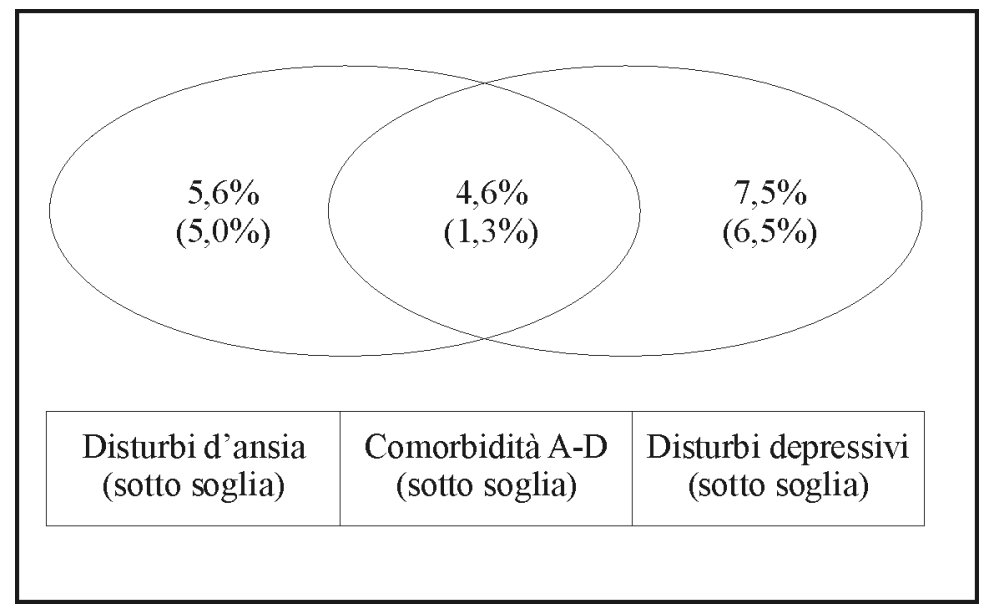

\section{Figura 1}

Prevalenza dei disturbi d'ansia e depressivi nella medicina di primo livello secondo indagini dell'OMS. (64) del capitale umano.(70)

Sono stati inclusi sia i costi diretti sia i costi indiretti. I costi diretti comprendevano la spesa farmaceutica, i costi dell' assistenza psichiatrica ed i costi dell'assistenza medica non psichiatrica. I costi indiretti includevano i costi dovuti alla perdita di anni di vita per suicidio ed i costi per ridotta produttività lavorativa, per assenteismo e per scarso rendimento sul lavoro a causa del disturbo psichiatrico.

Il costo annuale di malattia del disturbo d'ansia è stato stimato per gli Stati Uniti in circa 42,3 miliardi di dollari al valore del 1990, o di 63,1 miliardi di dollari al valore del 1998. Ogni anno il singolo paziente affetto da disturbo d'ansia induce un costo sociale totale pari a $\$ 1542$, al valore del 1990; il costo medio annuale pro capite per perdita di produttività lavorativa è di circa \$256, dei quali 1'88\% è dovuto a scarso rendimento sul lavoro e non ad assenteismo.

La figura 2 riporta la distribuzione percentuale dei principali costi diretti ed indiretti che contribuiscono a determinare il costo di malattia del disturbo d'ansia. La spesa farmaceutica ammonta ad appena il $2 \%$ del costo totale di malattia stimato negli USA. La principale voce di costo è rappresentata dall' assistenza sanitaria non psichiatrica (54\%); al secondo posto figura il costo dell' assistenza psichiatrica (31\%). L'ospedalizzazione rappresenta poco meno del $15 \%$ del costo totale del disturbo d'ansia. I pazienti che soffrono di disturbi d'ansia, in confronto con quelli che non ne soffrono, tendono a consumare maggiori quantità di servizi psichiatrici, ma non di visiste mediche.

Complessivamente i costi diretti ammontano all' $87 \%$ del costo totale di malattia del disturbo d'ansia, mentre i costi indiretti ammontano solo al $13 \%$ circa. Il costo indiretto dovuto alla mortalità per suicidio è stato stimato in circa il 3\% del costo totale, mentre il costo indiretto per perdita di produttività in ambito lavorativo è pari a circa il $10 \%$ del costo totale di malattia. L'assenteismo tende ad aumentare nei soggetti affetti da disturbi d'ansia ma non in modo clamoroso, mentre il disturbo riduce il rendimento sul posto di lavoro.

Il costo di malattia stimato da Greenberg (42,3 miliardi di dollari) è simile in valore totale a quello stimato da Dupont e collaboratori (46,6 miliardi di dollari), ma differente per la distribuzione dei costi (71). Nello studio di Dupont, infatti, (vedi tabella 2) i costi indiretti ammontano a circa il $77 \%$ del totale: il differente risultato dipende verosimilmente dal fatto che Dupont nella sua analisi ha sottostimato i costi diretti dell'assistenza sanitaria psichiatrica e non psichiatrica.

I costi di malattia del disturbo d'ansia sono dello stesso ordine di grandezza dei costi della depressione stimati per gli Stati Uniti sempre 


\begin{tabular}{|ccccc|}
\hline & $\begin{array}{c}\text { Costo di Tutte le } \\
\text { Malattie Mentali }\end{array}$ & $\begin{array}{c}\text { Costo di Malattia } \\
\text { dei Disturbi d'Ansia }\end{array}$ & $\begin{array}{c}\text { Incidenza dei disturbi d'ansia } \\
\text { sul costo totale } \\
\text { delle malattie mentali }\end{array}$ \\
\hline Costi Diretti & 67,0 & 10,7 & $23,1 \%$ & $\%$ \\
\hline Costi Indiretti & & & & $16,0 \%$ \\
\hline Morbilità & 63,1 & 34,2 & $73,4 \%$ & $54,2 \%$ \\
\hline Mortalità & 11,8 & 1,3 & $2,7 \%$ & $10,8 \%$ \\
\hline Altri costi & 6,0 & 0,4 & $0,8 \%$ & $6,2 \%$ \\
\hline TOTALE & $\mathbf{1 4 7 , 8}$ & $\mathbf{4 6 , 6}$ & $\mathbf{1 0 0 , 0} \%$ & $\mathbf{3 1 , 5 \%}$ \\
\hline
\end{tabular}

Tabella 2

Stima e struttura dei costi di malattia dei disturbi d'ansia in confronto con il costo sociale di tutte la malattie mentali negli Stati Uniti, al valore del 1990, secondo Dupont. (71) da Greenberg e collaboratori, ma differiscono per distribuzione delle voci di costo.(72)

Il costo di malattia della depressione negli USA è stato stimato in oltre 52 miliardi di dollari/anno, corrispondente a circa 3.000 dollari/ anno per ogni paziente, al valore del 1990.

Il costo totale della depressione è costituito per circa un quarto dai costi diretti sanitari e per i rimanenti tre quarti dai costi indiretti.

I costi indiretti sono costituiti per il $60 \%$ da perdita di produttività per assenteismo dal lavoro, per il $21 \%$ da scarso rendimento sul lavoro, e per il restante $19 \%$ dalla perdita di produttività dovuta a morti per suicidio.

La spesa farmaceutica rappresenta solo il $12 \%$ circa del totale dei costi diretti, ossia circa il 2,8\% del costo totale di malattia, una quota percentuale molto simile a quella stimata per il costo dell'ansia. I costi per ricoveri nel caso della depressione rappresentano circa il $57 \%$ dei costi diretti sanitari, ossia poco più del $13 \%$ del costo totale di malattia. La quota percentuale della spesa ospedaliera è sensibilmente più elevata nel caso del disturbo d'ansia.

D'altra parte si deve osservare come la stima del costo della depressione effettuata da Greenberg fosse conservativa perchè non includeva le depressioni sottosoglia e le depressioni atipiche, non considerava i costi dovuti all'eccesso di ospedalizzazioni per malattie non psichiatriche indotto dalla depressione, o i costi della diagnostica richiesta impropriamente dai soggetti depressi che presentavano sintomi somatici.

I pazienti affetti da disturbo d'ansia e quelli affetti da disturbo depressivo inducono costi più elevati nel settore dell' assistenza sanitaria di primo livello (Medicina Generale) (73)

In un campione di soggetti seguiti per sei mesi dal servizio sanitario di primo livello degli Stati Uniti, il costo medio per paziente affetto da depressione o da disturbo d'ansia secondo
DSM-III-R era di \$ $2.390 \pm 4.168$, quello medio per pazienti affetti da depressione o da ansia sottosoglia era di \$ $1.098 \pm 1.359$, mentre quello dei soggetti senza ansia o depressione era di $\$ 1.397 \pm 2.847$. Le differenze permanevano ampie dopo aggiustamento dei costi in funzione delle comorbilità. Le differenze dei costi erano dovute alla maggiore utilizzazione di servizi assistenziali non psichiatrici da parte sia del paziente depresso sia del paziente ansioso.(73)

Nel caso di pazienti affetti da disturbo d'ansia generalizzata, la gravità dei sintomi, la storia di precedenti episodi di disturbo d'ansia, l'età senile e la presenza di comorbilità sono condizioni che giocano un ruolo principale nell'indurre un significativo aumento dei costi sanitari e dei costi totali di malattia. (74)

In uno studio condotto su oltre 1.000 pazienti affetti da disturbo d'ansia generalizzata i soggetti che presentavano comorbilità organiche e psichiatriche inducevano in tre mesi un costo medio totale di $\$ 1.208$, mentre quelli che

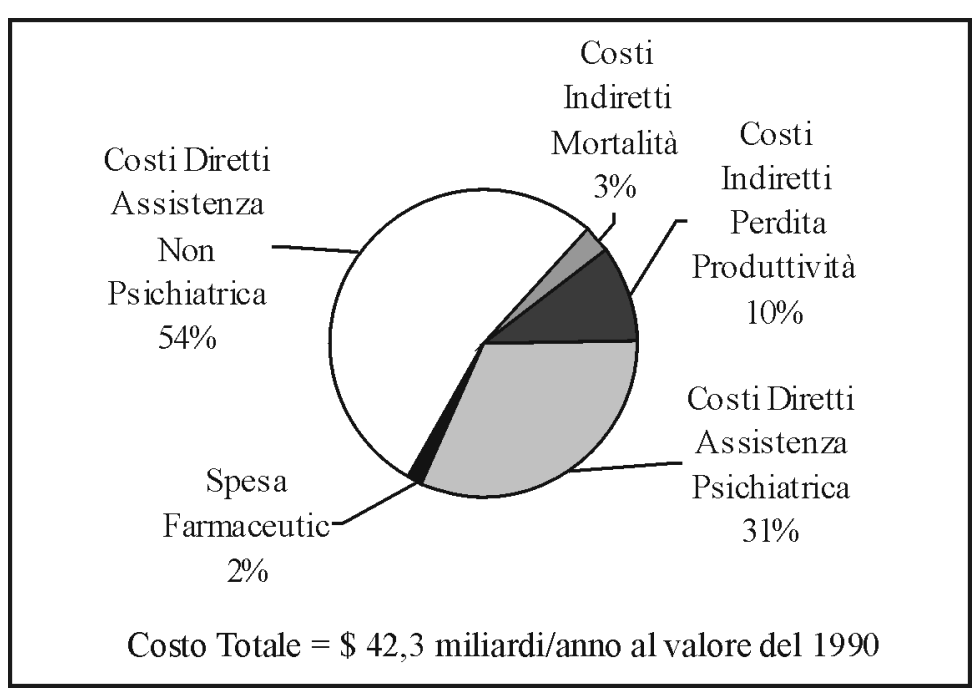

Figura 2

Distribuzione percentuale dei costi di malattia dei disturbi d'ansia negli Stati Uniti, secondo Greenberg.(70) 
non avevano comorbilità inducevano un costo significativamente inferiore stimato in appena $\$ 733$ (vedi tabella 3).

In valore assoluto le differenze più consistenti riguardavano i costi per ricoveri ospedalieri, i costi per assistenza psichiatrica e per assistenza medica, i costi per esami di laboratorio e per farmaci, nonchè $i$ costi indiretti dovuti all'assenteismo nei soggetti in attività produttiva.

Indipendentemente dalla presenza di comorbilità, il costo per ricoveri ed il costo indiretto per perdita di produttività erano i due maggiori fattori di costo del disturbo d'ansia generalizzata, mentre il costo per farmaci rappresentava una componente minima, inferiore al $3 \%$ del costo totale di malattia. (74)

In uno studio francese, condotto secondo la prospettiva dell'Assicurazione Malattie, la gravità del disturbo d'ansia generalizzata è stata correlata con un significativo incremento dei costi totali di malattia. (75) L'incremento dei costi era dovuto principalmente alla maggiore necessità di assistenza ospedaliera ed alla maggiore quantità di giorni lavorativi persi da parte dei soggetti più disturbati (vedi figura 3 ).

\section{Costi sanitari \\ del disturbo da attacchi di panico}

Il disturbo da attacchi di panico induce un consumo elevato, molto variabile, di risorse sanitarie sia per assistenza psichiatrica che per assistenza sanitaria di base. $(76,77)$

Un'analisi dei costi, condotta su un campione di 391 pazienti statunitensi affetti da attacchi di panico ed arruolati in trials clinici di Fase $\mathrm{I}^{\circ}$ e $\mathrm{II}^{\circ}$, ha evidenziato che il $70 \%$ dei pazienti utilizzava servizi sanitari di primo livello, il $47 \%$ utilizzava i servizi psichiatrici territoriali, il 46,8\% i servizi specialistici non psichiatrici ed il $20,6 \%$ i servizi cardiologici. I soggetti che denunciavano un maggior numero di attacchi di panico ricorrevano più frequentemente all'assistenza sanitaria di primo livello, mentre i pazienti più gravi e maggiormente disturbati utilizzavano con maggiore frequenza l'assistenza specialistica psichiatrica. (77)

I costi medi/paziente per episodio e per anno sono riportati nella tabella 4 , suddivisi per tipo di servizio consumato. Ogni episodio di attacco di panico negli USA costava circa 3.339 dollari, al valore del 1993, metà dei quali circa erano spesi per l'assistenza specialistica psichiatrica.

La variabilità interindividuale della spesa sanitaria era molto elevata tra i pazienti affetti da attacchi di panico: il costo episodio/paziente variava da $\$ 0$ a $\$ 27.728$ : un certo numero di soggetti non consumava risorse sanitarie mentre un $10 \%$ dei soggetti erano forti consumatori e spendevano circa il $45 \%$ del totale delle risorse consumate dal campione.(77)

I soggetti affetti da disturbi da attacchi di panico frequentemente presentano sintomi
Tabella 3

Costi diretti ed indiretti associati al disturbo d'ansia generalizzata. (74)

\begin{tabular}{|lccc|}
\hline \multicolumn{1}{|c}{ Voci di costo } & Costo Medio/Paziente GAD/3 mesi (\$U.S.) & P \\
Con comorbilità & Senza comorbilità & \\
$(\mathbf{n = 6 0 4 )}$ & & $395)$ & \\
\hline Costi Diretti Sanitari & 423 & 158 & $<0,01$ \\
\hline Ricoveri ospedalieri & 104 & 62 & NS \\
\hline Pronto Soccorso e Chirurgia & 187 & 55 & $<0,05$ \\
\hline Assistenza Psichiatrica & 131 & 35 & $<0,05$ \\
\hline Assistenza in Medicina Interna & 2 & 5 & NS \\
\hline Rianimazione & 44 & 19 & $<0,01$ \\
\hline Diagnostica e laboratorio & 335 & 308 & $\mathrm{NS}$ \\
\hline Assistenza extraospedaliera & 43 & 25 & $<0,001$ \\
\hline Farmaci & & 733 & $<0,001$ \\
\hline Costi Indiretti & 416 & & $<0,01$ \\
\hline Perdita produttività lavorativa & $\mathbf{1 2 0 8}$ & & \\
\hline Costi Totali & & 243 & \\
\hline
\end{tabular}




\begin{tabular}{|c|c|c|c|c|}
\hline & \multicolumn{4}{|c|}{ Costo Medio/Paziente con Disturbo da Attacchi di Panico (1993) } \\
\hline & \multicolumn{2}{|c|}{ Costo/Episodio (\$U.S.) } & \multicolumn{2}{|c|}{ Costo Annuale (\$U.S.) } \\
\hline & Media & $\mathrm{SD}$ & Media & SD \\
\hline \multicolumn{5}{|c|}{ Servizi Non Psichiatrici } \\
\hline Cardiologia & 117 & 688 & 26 & 108 \\
\hline Neurologia & 46 & 194 & 16 & 113 \\
\hline Altri Specialità & 444 & 1529 & 156 & 884 \\
\hline Medicina Generale & 1012 & 1972 & 264 & 834 \\
\hline Servizi Psichiatrici & 1725 & 3147 & 483 & 1354 \\
\hline Totale & 3339 & 4880 & 969 & 2045 \\
\hline
\end{tabular}

Tabella 4

Costi medi stimati per l'utilizzo di servizi sanitari extraospedalieri da parte di pazienti affetti da disturbo da attacchi di panico.(77) somatici, spesso molto forti ed invalidanti, ed in alta percentuale sono affetti anche da altre patologie psichiatriche ed organiche associate. Per questi motivi il paziente affetto da disturbo di panico trascorre un lungo periodo di malattia prima di essere correttamente diagnosticato e curato, induce elevati consumi di servizi sanitari territoriali ed ospedalieri e significative perdite economiche al sistema produttivo. Il consumo improprio di risorse sanitarie e le consistenti perdite di produttività potrebbero essere significativamente ridotte o azzerate, qualora il disturbo di panico venisse gestito in modo adeguato ed efficace.

Un corretto trattamento può essere efficace nel 70-90\% dei pazienti affetti da disturbi di panico ed un trattamento precoce evita la progressione della malattia e lo sviluppo dell'agorafobia.

La gestione del paziente affetto da disturbi di attacchi di panico può essere effettuata dal medico di Medicina Generale, ma spesso richiede il supporto dello psichiatra sia per affrontare problemi diagnostici difficili sia per effettuare psicoterapie cognitivo-comportamentali.

Trattare correttamente un paziente con disturbi di panico comporta costi aggiuntivi specifici, dovuti ai farmaci, alla consulenza psichiatrica ed all'eventuale psicoterapia. Tali costi vanno esaminati sia in relazione al miglioramento della qualità di vita del paziente sia in relazione ai risparmi inducibili a vantaggio del SSN, per la riduzione o l'azzeramento del consumo improprio di risorse sanitarie, ed a vantaggio della società, per il ristabilimento di una normale attività produttiva e la riduzione complessiva dei costi di malattia.

La modificazione dei costi diretti ed indiretti del disturbo di panico indotta dalla presa in carico del paziente da parte di un servizio psichiatrico è stato esaminato da un gruppo di ricercatori spagnoli. (78) Il consumo di risorse è stato rilevato in un gruppo di 61 pazienti affetti da attacchi di panico durante un anno precedente ed un anno successivo alla presa in carico da parte di un nuovo servizio ambulatoriale psichiatrico territoriale operante a Barcellona. I principali risultati sono riportati nella tabella 5 .

Il trattamento attuato dal servizio psichiatrico è stato nella quasi totalità dei casi di tipo farmacologico, basato prevalentemente sull'uso di alprazolam e/o di clomipramina. Ciò ha comportato un aumento medio di $140 \$$ nella spesa farmaceutica annuale per paziente. Ben più consistente è risultato l'incremento dei costi dovuti alle visite psichiatriche, passate da $24 \$$ dell'anno precedente a $482 \$$ nel primo anno di presa in carico del paziente da parte del servizio psichiatrico. Questi aumenti dei costi sanitari sono stati in parte controbilanciati da consistenti riduzioni del costo per visite mediche non psichiatriche, per esami di laboratorio, e dall' azzeramento del costo ospedaliero del co-

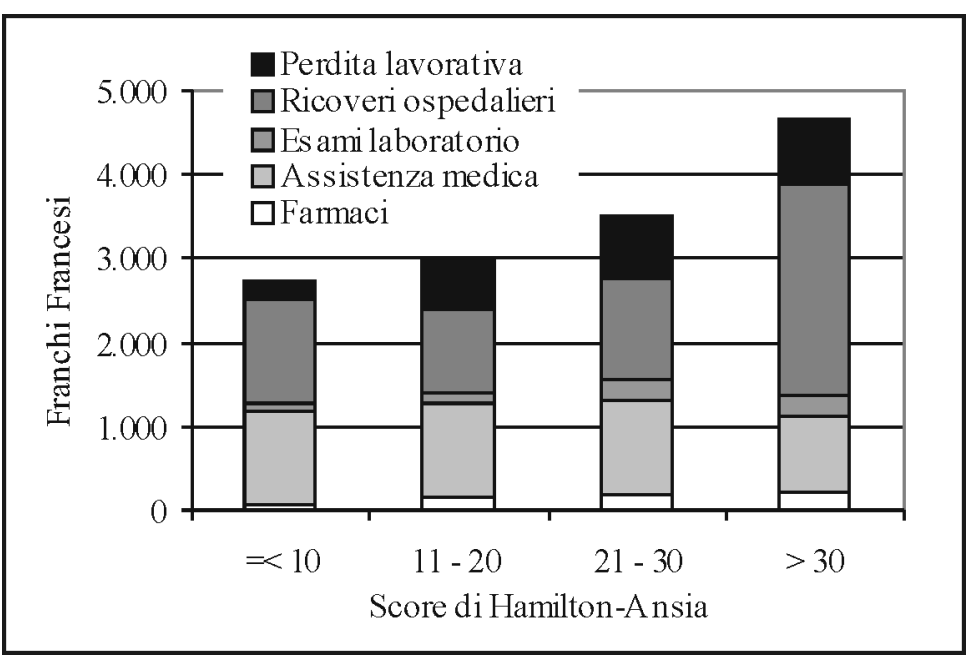

\section{Figura 3}

Costo medio trimestrale/paziente affetto da ansia generalizzata in funzione della gravità del disturbo, secondo la prospettiva dell'Assicurazione Malattie di Francia. (75) 
sto per medicine alternative. Il bilancio finale dei costi diretti sanitari vede un incremento annuale medio di $280 \$$ per paziente trattato. $\mathrm{Si}$ tratta di un costo incrementale modesto ampiamente giustificabile se confrontato col risultato terapeutico ottenuto, assai soddisfacente in termini di riduzione del numero di attacchi di panico e di episodi di agorafobia, quasi totalmente eliminati, ed in termini di qualità di vita $\mathrm{e}$ di ripresa funzionale, nettamente migliorate.

Se consideriamo il risultato nella prospettiva più ampia della società, la nuova strategia terapeutica, basata sulla presa in carico del paziente affetto da disturbo di panico da parte di un servizio psichiatrico territoriale, è risultata oltremodo conveniente perchè ha comportato un risparmio netto annuale di circa $600 \$$ per ogni soggetto trattato. Infatti, i costi indiretti annuali per paziente, dovuti al disturbo di panico, sono diminuiti da $1.076 \$$ a $228 \$$, con un risparmio annuale netto sulle perdite di produttività di $849 \$$ per paziente trattato. (78)

Questo lavoro evidenzia come sia possibile curare con buona efficacia ed efficienza il disturbo di panico, con risultati compatibili con le esigenze di bilancio del SSN e con grande beneficio netto per la società. Condizione essenziale risulta essere la possibilità di attuare tempestivamente una corretta diagnosi di disturbo di panico ed impostare un'adeguata terapia personalizzata. I risultati ottenuti dai ricercatori spagnoli possono essere trasferiti in linea generale anche all' attuale situazione italiana. Si deve notare come in Italia la capillare rete di medici di Medicina Generale presente sul territorio, qualora attivata correttamente in una rete collaborativa coi servizi psichiatrici, potrebbe contribuire a ridurre sensibilmente il costo sanitario rispetto a quanto stimato in Spagna.

\section{Valutazione farmacoeconomica delle benzodiazepine}

Le stime del costo di malattia dimostrano che il disturbo d'ansia ha un grande impatto sociale ed ogni anno assorbe una rilevante quantità di risorse sanitarie ed extrasanitarie.(70)

I disturbi d'ansia peggiorano la qualità della vita, favoriscono il manifestarsi di forme più o meno gravi di depressione e di abuso di sostanze, inducono un aumento significativo della domanda di assistenza sanitaria per sintomi somatici, ma soprattutto comportano un aumento dei ricoveri ospedalieri ed un aumento di consumo improprio di molti altri servizi sanitari.

La stima del costo di malattia prodotta da Greenberg era basata sul riscontro epidemiologico secondo il quale, negli Stati Uniti agli inizi degli anni '90, solo il $27 \%$ dei soggetti ai quali era stato diagnosticato un disturbo d'ansia aveva ricevuto un trattamento.(79) Inoltre, è verosimile che molti dei soggetti trattati non abbiano ricevuto una terapia adeguata ed efficiente.

Al pari della depressione, anche il disturbo d'ansia sembra essere ancora misconosciuto, poco frequentemente diagnosticato e soprattutto troppo sovente sottotrattato, non trattato affatto o curato con terapie inadeguate.

Sembra plausibile ritenere che l'elevato co-
Tabella 5

Costi annuali medi diretti, indiretti e totali per paziente affetto da attacchi di panico prima e dopo la presa in carico da parte del servizio psichiatrico spagnolo. Dati in parte rielaborati dal lavoro di Salvador-Carrula et al. (78)

\begin{tabular}{|lccc|}
\hline & $\begin{array}{c}\text { Anno Pre-Trattamento } \\
\text { Psichiatrico }\end{array}$ & $\begin{array}{c}\text { Primo Anno di } \\
\text { Trattamento Psichiatrico }\end{array}$ & Differenza \\
\hline Costi diretti (\$) & & 11 & -181 \\
\hline $\begin{array}{l}\text { Visite mediche non } \\
\text { psichiatriche (\$) }\end{array}$ & 192 & 482 & +458 \\
\hline Visite psichiatriche (\$) & 24 & 0 & -62 \\
\hline Ricoveri ospedalieri (\$) & 62 & 73 & -73 \\
\hline Esami di laboratorio (\$) & 147 & 191 & +140 \\
\hline Farmaci (\$) & 51 & 0 & -2 \\
\hline Medicine alternative (\$) & 2 & 758 & +280 \\
\hline Totale Costi Diretti (\$) & 478 & 228 & -849 \\
\hline Costi indiretti (\$) & 1076 & $\mathbf{9 8 5}$ & $\mathbf{- 5 6 9}$ \\
\hline $\begin{array}{l}\text { Costi totali } \\
\text { attacchi di panico (\$) }\end{array}$ & $\mathbf{1 5 5 4}$ & & \\
\hline
\end{tabular}


sto sociale del disturbo d'ansia possa essere ridotto sensibilmente mediante interventi terapeutici efficaci. In particolare è molto probabile che l'aumento della spesa farmaceutica, che si indurrebbe con l'allargamento del numero di pazienti trattati con benzodiazepine, sarebbe ampiamente compensato dai risparmi sui consumi di risorse sanitarie indotte dal disturbo d'ansia.(70)

Tuttavia, le benzodiazepine, benchè dotate di un eccellente margine di sicurezza, non sono prive di qualche inconveniente, come la possibilità di indurre abitudine e tolleranza, di manifestare reazioni avverse soprattutto in associazione con altri farmaci, e di non essere totalmente efficaci in un certo numero di pazienti affetti da disturbo d'ansia.

Per decidere sulla convenienza ad usare le benzodiazepine in malattie ad elevato impatto sociale come il disturbo d'ansia, gli attacchi di panico e le sindromi miste ansioso-depressive, occorrerebbe analizzare accuratamente i costi ed i benefici, valorizzando questi ultimi in termini monetari.

Una tecnica che evita questo passaggio è l'analisi costo/efficacia, tecnica considerata fondamentale per scegliere l'alternativa terapeutica più conveniente tra quelle disponibili.

Tuttavia, anche l'analisi costo/efficacia presenta alcuni limiti semantici, pratici e concettuali.(80) Infatti, spesso questo tipo di analisi è usata in modo inappropriato ed i risultati sono mal interpretati; inoltre, nel condurre un' analisi costo/efficacia i costi ed i risultati clinici considerati variano in funzione del decisore, e quindi anche le conclusioni possono variare in relazione alla prospettiva dell' analisi; infine, l' analisi costo/efficacia indica la soluzione più efficiente ma non necessariamente quella più rispettosa di altri valori (es di equità) o di opzioni soggettive degli attori coinvolti: pazienti, medici, terzi paganti, ecc.

\section{“Analisi del valore” delle benzodiazepine}

Per superare queste difficoltà e tener conto della complessità dei fattori che possono determinare una decisione in campo sanitario alcuni autori utilizzano una tecnica denominata “analisi dei valori”.(81)

Tale tecnica di analisi decisionale consiste nel formulare e testare un insieme di valori proposti che variano in funzione del tipo di outcome clinico ed economico ed in funzione delle diverse prospettive dei decisori coinvolti $o$ attori interessati alla decisione.

Il presupposto è che un outcome positivo o negativo per un paziente potrebbe avere un significato molto differente per il medico, per l'ospedale, per il SSN o per altri decisori.(82)

Secondo alcuni autori per analizzare la convenienza ad usare le benzodiazepine in accordo con la tecnica "analisi dei valori" è necessario considerare la prospettiva di almeno cinque diversi attori-decisori: il paziente, il medico, l'assicurazione, l'azienda farmaceutica e la società.(80)

Questa scelta rispecchia la struttura del sistema assistenziale degli Stati Uniti dove l'assicurazione è il terzo pagante: in Italia, data la struttura del nostro Welfare State, è opportuno sostituire questo decisore con il SSN.

Considerando di dover analizzare il valore, positivo e negativo, attribuito da ognuno dei decisori all'outcome sanitario ed all'outcome economico di ciascuna alternativa, è utile costruire una matrice, come quella rappresentata nella tabella 6. Per ogni decisore considerato (abbiamo eliminato la prospettiva dell'industria farmaceutica perchè non rilevante ai fini della presente discussione), le quattro caselle contengono il giudizio di valore relativo all' incrocio tra tipo di outcome, sanitario ed economico, e segno positivo o negativo dell'outcome stesso: questi giudizi possono essere ottenuti in vario modo, ad esempio analizzando esaustivamente la letteratura disponibile.

La tabella 6 riporta i giudizi di valore proposti da Lyons e collaboratori circa l'uso delle benzodiazepine in generale. (80) Nella stessa tabella, in corsivo, vengono anche proposte le considerazioni di merito che riteniamo di poter ricavare dall' analisi della letteratura a sostegno della opportunità e convenienza ad utilizzare alprazolam nel trattamento dei disturbi d'ansia, del disturbo di panico e della sindrome mista ansioso-depressiva in Italia.

Secondo Lyons e collaboratori le benzodiazepine risultano molto utili per ognuna delle prospettive considerate. Infatti le benzodiazepine hanno un importante ruolo nel sistema sanitario e risultano efficaci e sicure quando usate in modo appropriato per gestire $\mathrm{i}$ disturbi d'ansia. Tuttavia l'analisi evidenzia anche come questi farmaci possono avere un valore negativo per ognuna delle prospettive, quando siano usate in modo inappropriato, ossia al di fuori delle indicazioni validate, per periodi di tempo diversi da quelli consigliati, in pazienti a rischio di suicidio, senza un'attenta strategia per sospendere il trattamento.

In base alla sintesi delle caratteristiche farmacologiche e terapeutiche sopra riportate, l'uso di alprazolam nelle indicazioni terapeutiche approvate presenta più vantaggi che svantaggi per ognuno dei quattro decisori considerati. Questo giudizio tiene conto sia del valore 
terapeutico assoluto che di quello relativo alle alternative disponibili e raccomandabili per ognuna delle indicazioni ammesse.

Per quanto riguarda $i$ costi ed un giudizio più complesso di costo/efficacia, occorre innanzitutto considerare che in Italia il costo d'acquisto di alprazolam, come quello delle altre benzodiazepine, è a totale carico del paziente. Questa scelta di politica sanitaria discrimina, evidentemente penalizzandoli sul piano eco- nomico, i pazienti affetti da disturbo d'ansia o da disturbo di panico da quelli affetti da disturbo depressivo. Infatti, l'inclusione delle benzodiazepine nella classe $\mathrm{C}$ (farmaci non rimborsabili dal SSN), deve essere interpretata come conseguenza di una valutazione tecnica che ha portato a ritenere di secondaria rilevanza il disturbo d'ansia ed il disturbo di panico, ovvero a concludere che il rapporto rischio/beneficio e costo/beneficio delle benzodiazepine in

\begin{tabular}{|c|c|c|}
\hline \multicolumn{3}{|c|}{ Prospettiva del paziente } \\
\hline Valore & Positivo & Negativo \\
\hline Clinico & $\begin{array}{l}\text { Riduzione, controllo dei sintomi. } \\
\text { Con alprazolam si ha un rapido } \\
\text { miglioramento dei disturbi d'ansia, } \\
\text { del disturbo di panico, semplice o } \\
\text { complicato da agorafobia, e del } \\
\text { disturbo depressivo con ansia. }\end{array}$ & $\begin{array}{l}\text { Dipendenza, reazioni avverse, incidenti. } \\
\text { Le reazioni avverse ad alprazolam e } \\
\text { gli eventuali incidenti d'uso sono } \\
\text { simili a quelle di tutte le } \\
\text { benzodiazepine. } \\
\text { La dipendenza grave è un fenomeno } \\
\text { non frequente con alprazolam, se } \\
\text { viene usato secondo indicazioni. }\end{array}$ \\
\hline Economico & $\begin{array}{l}\text { Buona efficienza, basso costo unitario, } \\
\text { miglioramento performance lavorativa. } \\
\text { Alprazolam, rispetto ad altri farmaci, } \\
\text { offre maggiori garanzie di efficacia } \\
\text { ed efficienza quando usata per il } \\
\text { disturbo di panico e per la } \\
\text { depressione mista ad ansia. Il } \\
\text { paziente deve sopportare il costo } \\
\text { d'acquisto della benzodiazepina, ma } \\
\text { questo viene ampiamente annullato } \\
\text { dal recupero delle possibilità } \\
\text { lavorative e dal miglioramento della } \\
\text { qualità di vita. }\end{array}$ & $\begin{array}{l}\text { Necessità di visite multiple, costi elevati } \\
\text { per trattamenti prolungati, costi per } \\
\text { eventuali incidenti d'uso e per } \\
\text { dipendenza. } \\
\text { Sono costi spesso più legati alla } \\
\text { patologia che al tipo di farmaco: } \\
\text { alprazolam non è differente da altre } \\
\text { benzodiazepine e da altri } \\
\text { antidepressivi. }\end{array}$ \\
\hline \multicolumn{3}{|c|}{ Prospettiva del medico } \\
\hline Valore & Positivo & Negativo \\
\hline Clinico & $\begin{array}{l}\text { Soddisfazione del paziente, sollievo dei } \\
\text { sintomi, indicazioni multiple e facilità } \\
\text { d'uso. } \\
\text { Alprazolam, per profilo di efficacia e } \\
\text { di tollerabilità, rappresenta una delle } \\
\text { poche benzodiazepine orali che } \\
\text { facilitano la prescrizione del medico, } \\
\text { garantendogli la gratitudine del } \\
\text { paziente. }\end{array}$ & $\begin{array}{l}\text { Gestione complessa, difficile la } \\
\text { sospensione, eventuale tolleranza, } \\
\text { reazioni avverse ed intossicazioni. } \\
\text { La sospensione dopo trattamenti } \\
\text { prolungati può essere difficoltosa. } \\
\text { La tolleranza ad alprazolam non è } \\
\text { stata dimostrata e rari sono i casi di } \\
\text { reazioni avverse che comportano la } \\
\text { sospensione del trattamento. }\end{array}$ \\
\hline Economico & $\begin{array}{l}\text { Prescrizione efficiente, fatturabile, e con } \\
\text { opportunità di molteplici visite. }\end{array}$ & $\begin{array}{l}\text { Costi per eventuali controversie } \\
\text { giudiziarie. } \\
\text { In Italia i casi di controversie } \\
\text { giudiziarie riguardanti l'uso di } \\
\text { benzodiazepine sono rari. } \\
\text { Il medico può avere difficoltà a } \\
\text { prescrivere alprazolam perchè, come } \\
\text { tutte le benzodiazepine orali, è un } \\
\text { prodotto in fascia } C \text {, non } \\
\text { rimborsabile. }\end{array}$ \\
\hline
\end{tabular}

Tabella 6

"Matrice dei Valori" per giudicare la convenienza ad usare le benzodiazepine secondo Lyons e collaboratori.(80) Le considerazioni specifiche su alprazolam, in corsivo sono dell'autore e si riferiscono all'attuale situazione italiana. miglioramento performance lavorativa. Alprazolam, rispetto ad altri farmaci, offre maggiori garanzie di efficacia ed efficienza quando usata per il 政 d'acquisto della benzodiazepina, ma questo viene ampiamente annullato lavorative e dal miglioramento della qualità di vita.
Dipendenza, reazioni avverse, incidenti. Le reazioni avverse ad alprazolam e simili a quelle di tutte le benzodiazepine. on frequente con alprazolam, se

Necessità di visite multiple, costi elevati per trattamenti prolungati, costi per eventuali incidenti d'uso e per dipendenza.

Sono costi spesso piu legati alla armaco benzodiazepine e da altri antidepressivi. 


\begin{tabular}{|c|c|c|}
\hline \multicolumn{3}{|c|}{ Prospettiva del terzo pagante (SSN) } \\
\hline Valore & Positivo & Negativo \\
\hline Clinico & $\begin{array}{l}\text { Soddisfazione del cliente. } \\
\text { Alprazolam in un'alta percentuale di } \\
\text { casi risolve i difficili problemi del } \\
\text { disturbo di panico, semplice o } \\
\text { complicato, del disturbo d'ansia e } \\
\text { della depressione mista ad ansia. }\end{array}$ & $\begin{array}{l}\text { Insoddisfazione del cliente. } \\
\text { Alprazolam in un numero limitato di } \\
\text { casi non risulta efficace o non } \\
\text { tollerato alle dosi indicate nel } \\
\text { trattamento del disturbo di panico, } \\
\text { del disturbo d'ansia e della } \\
\text { depressione mista ad ansia. }\end{array}$ \\
\hline Economico & $\begin{array}{l}\text { Basso costo unitario. } \\
\text { In Italia il costo d'acquisto di } \\
\text { alprazolam è a carico del paziente. } \\
\text { Il SSN ne ricava un beneficio netto } \\
\text { elevato perchè non ha spese } \\
\text { farmaceutiche su patologie di grande } \\
\text { impatto sociale e risparmia elevate } \\
\text { quantità di risorse sanitarie non più } \\
\text { utilizzate dai pazienti curati con } \\
\text { alprazolam. L'uso di alprazolam può } \\
\text { consentire un risparmio sulla spesa } \\
\text { farmaceutica degli antidepressivi } \\
\text { usati impropriamente come } \\
\text { ansiolitici. }\end{array}$ & $\begin{array}{l}\text { Alta utilizzazione. } \\
\text { In Italia l'elevata frequenza di } \\
\text { utilizzazione di alprazolam non pesa } \\
\text { negativamente sul SSN perchè il } \\
\text { farmaco non viene rimborsato. } \\
\text { Possono aumentare alcune voci di } \\
\text { costo a carico del SSN (es. visite } \\
\text { specialistiche) ma diminuiscono } i \\
\text { costi per esami di laboratorio e per } \\
\text { ricoveri. }\end{array}$ \\
\hline \multicolumn{3}{|c|}{ Prospettiva della società } \\
\hline Valore & Positivo & Negativo \\
\hline Clinico & $\begin{array}{l}\text { Miglioramento della salute e del } \\
\text { benessere dei cittadini. } \\
\text { Alprazolam è altamente efficace ed } \\
\text { efficiente nel trattamento di tre } \\
\text { grandi problemi sanitari a forte } \\
\text { impatto sociale: il disturbo d'ansia, il } \\
\text { disturbo da attacchi di panico ed il } \\
\text { disturbo depressivo misto ad ansia. } \\
\text { Migliora, quindi la qualità di vita dei } \\
\text { pazienti e dei loro familiari. }\end{array}$ & $\begin{array}{l}\text { Reazioni avverse, incidenti d'uso, } \\
\text { dipendenza, paure e preoccupazioni. } \\
\text { Alprazolam, come le altre } \\
\text { benzodiazepine, può indurre } \\
\text { fenomeni di abitudine o di } \\
\text { dipendenza. } \\
\text { La possibilità di agire con farmaci su } \\
\text { fenomeni psichici genera ansie e } \\
\text { preoccupazioni, spesso su base } \\
\text { ideologica più che fattuale. }\end{array}$ \\
\hline Economico & $\begin{array}{l}\text { Crea posti di lavoro. Bassi costi per } \\
\text { unità. } \\
\text { La cura dei disturbi d'ansia, di } \\
\text { panico e delle depressioni riduce le } \\
\text { perdite economiche nelle attività } \\
\text { produttive, aumenta le opportunità } \\
\text { di integrazione nel mondo lavorativo } \\
\text { e riduce il numero di suicidi. } \\
\text { Alprazolam può creare benefici netti } \\
\text { elevati per la società. }\end{array}$ & $\begin{array}{l}\text { Grande spesa totale. } \\
\text { La spesa sociale di malattia } \\
\text { correlabile al disturbo d'ansia, al } \\
\text { disturbo di panico ed alla } \\
\text { depressione viene nettamente ridotta } \\
\text { in valore assoluto sebbene aumentino } \\
\text { i costi di trattamento per l'assistenza } \\
\text { farmaceutica e medica. }\end{array}$ \\
\hline
\end{tabular}

Tabella 6 (continua) tali indicazioni è inferiore a quello degli antidepressivi, che invece sono rimborsati.

Si deve innanzitutto osservare come non tutti gli antidepressivi, attualmente inclusi nella classe A, siano stati approvati per il trattamento del disturbo di panico e come nessuno sia approvato come ansiolitico. Tra gli antidepressivi maggiormente prescritti nell'ultimo anno (vedi tabella 7) la fluoxetina, la venlafaxina e la reboxetina non risultano approvate per l'indicazione "disturbo di panico".

E' noto che l'ansia è un sintomo frequentemente presente nel soggetto depresso e che gli antidepressivi sono in grado di controllare i sintomi d'ansia nel caso vi sia una depressione mista ad ansia: questo fatto porta a sottolineare come si debba privilegiare l'uso di un antidepressivo quando il soggetto sia affetto 
ALPRAZOLAM: profilo farmacoeconomico nei disturbi d'ansia e da attacchi di panico

\section{Tabella 7}

Distribuzione percentuale delle prescrizioni degli antidepressivi più venduti in Italia in funzione della diagnosi. (dati SPM $4^{\circ}$ QTR anno 2001 dicembregennaio)

\section{Tabella 8}

Costo di acquisto di alprazolam e degli antidepressivi maggiormente prescritti in Italia nel 2001 per un range di posologie maggiormente usate secondo i dati SPM. I costi sono basati sui prezzi al pubblico validi al marzo $2002 e$ considerano un ciclo standard di 8 settimane.

\begin{tabular}{|lcccccc|}
\hline & Paroxetina & Sertralina & Fluoxetina & Venlafaxina & Clomipramina & Reboxetina \\
\hline $\begin{array}{l}\text { Totale prescrizioni } \\
\text { (n x 1000) }\end{array}$ & 1.795 .000 & 1.323 .000 & 852.000 & 737.000 & 737.000 & 379.000 \\
\hline Diagnosi & $\%$ & $\%$ & $\%$ & $\%$ & $\%$ & $\%$ \\
\hline Episodio depressivo & 58,8 & 63,4 & 61,6 & 58,2 & 57,6 & 57,8 \\
\hline Disturbi d'ansia & 23,7 & 16,9 & 20,2 & 23,6 & 10 & 20,6 \\
\hline $\begin{array}{l}\text { Disturbo ossessivo } \\
\text { compulsivo }\end{array}$ & 5,5 & 6,5 & 6 & 6,7 & 5,9 & 9,8 \\
\hline Depressione ricorrente & 2,4 & 2,3 & 0,3 & 1,4 & 7,1 & \\
\hline Disturbo bipolare & 0,9 & 1,5 & 0,4 & 2 & 4 & 2,6 \\
\hline Disturbo ansia fobica & 0,2 & 0,6 & 0,5 & 2,3 & 3,5 & 0,8 \\
\hline Altre indicazioni & 8,5 & 8,8 & 11 & 5,8 & 11,9 & 8,4 \\
\hline
\end{tabular}

da depressione anche quando vi siano concomitanti disturbi d'ansia. Tuttavia questa indicazione delle attuali linee-guida della depressione non comporta che tutti i soggetti affetti da disturbo d'ansia o da disturbo di panico, e soprattutto quelli che presentano un disturbo puro senza comorbilità, debbano essere trattati con antidepressivi.

Escludendo dal rimborso le benzodiazepine, il SSN risparmia sulla spesa farmaceutica e carica invece l'onere dell' acquisto sul cittadino o su eventuali sistemi assicurativi privati.

D'altra parte, la scelta di rimborsare gli antidepressivi, compresi quelli più recenti, favorisce l'ampliamento non solo dell'uso appropriato degli antidepressivi, risultato utile e raccomandato, ma anche di un uso improprio, sostenendo inevitabilmente un fenomeno di conversione delle prescrizioni da farmaci non rimborsabili a farmaci rimborsabili con un risul- tato economico finale per il SSN assai discutibile. Infatti, se è vero che il SSN evita di coprire la spesa per le benzodiazepine, accollandolo al paziente, è altrettanto vero che, per effetto di conversione, si assume comunque un maggior onere con il rimborso degli antidepressivi moderni che mediamente sono più costosi delle benzodiazepine (vedi tabella 8).

La tabella 7 evidenzia come una percentuale elevata di prescrizioni degli antidepressivi più venduti in Italia nell'ultimo anno, circa il $20 \%$, sia correlata a diagnosi di disturbi d'ansia. In questa categoria vengono compresi anche i disturbi di panico. Tuttavia, è evidente che una significativa percentuale di prescrizioni degli antidepressivi sia tecnicamente "offlabel": questo è senza dubbio vero per gli antidepressivi non approvati per il disturbo di panico e per tutti i casi di disturbi d'ansia puri (ad eccezione dell'indicazione DOC).

\begin{tabular}{|c|c|c|c|c|}
\hline \multirow{3}{*}{ Alprazolam } & \multirow{3}{*}{$\begin{array}{c}\begin{array}{c}\text { Costo/mg al pubblico } \\
\text { (marzo 2002 - Euro) }\end{array} \\
0,565\end{array}$} & \multicolumn{2}{|c|}{$\begin{array}{c}\text { Dose media } \\
\text { giornaliera }(\mathrm{mg} / \mathrm{die})\end{array}$} & \multirow{3}{*}{$\begin{array}{c}\begin{array}{c}\text { Costo acquisto farmaco per ciclo } \\
\text { terape utico di } 8 \text { settimane (Euro) }\end{array} \\
\begin{array}{c}15,82 \\
60,12\end{array}\end{array}$} \\
\hline & & Min & 0.5 & \\
\hline & & Max & 1.9 & \\
\hline \multirow{2}{*}{ Paroxetina } & \multirow{2}{*}{0,0576} & Min & 20.6 & 66,45 \\
\hline & & Max & 22.0 & 70,96 \\
\hline \multirow{2}{*}{ Sertralina } & \multirow{2}{*}{0,026} & Min & 58.0 & 84,45 \\
\hline & & Max & 59.0 & 85,90 \\
\hline \multirow{2}{*}{ Fluoxetina } & \multirow{2}{*}{0,0424} & Min & 20.0 & 47,49 \\
\hline & & Max & 32.4 & 76,93 \\
\hline \multirow{2}{*}{ Venlafaxina } & \multirow{2}{*}{0,0187} & Min & 49.1 & 51,42 \\
\hline & & Max & 177.0 & 185,35 \\
\hline \multirow{2}{*}{ Clomipramina } & \multirow{2}{*}{0,0045} & Min & 18.4 & 4,64 \\
\hline & & Max & 111.7 & 28,15 \\
\hline \multirow{2}{*}{ Reboxetina } & \multirow{2}{*}{0,1343} & Min & 4.7 & 35,20 \\
\hline & & Max & 8.0 & 60,17 \\
\hline
\end{tabular}


Escludendo il problema della distribuzione degli oneri economici tra cittadino e SSN, l'uso di alprazolam nelle indicazioni approvate risulta altamente conveniente per la società perchè consente di ridurre il costo complessivo di malattia dei disturbi d'ansia, del disturbo di panico e del disturbo depressivo misto ad ansia. Nel caso specifico del disturbo d'ansia e del disturbo di panico le valutazioni internazionali indicano che la società, con l'uso di farmaci molto efficaci ed efficienti, come alprazolam, ricava un beneficio netto consistente dovuto soprattutto ad una drastica riduzione delle perdite produttive.

\section{CONCLUSIONI}

Alprazolam, al pari delle altre benzodiazepine più utilizzate, risulta molto efficace e sicuro nel trattamento dei disturbi d'an- sia, dei disturbi da attacchi di panico ed anche nella sindrome mista ansioso-depressiva. I vantaggi relativi di alprazolam rispetto alle altre benzodiazepine sono la sua potenza, la sua notevole efficacia negli attacchi di panico e la sua peculiare attività sul tono dell'umore. Condizioni essenziali per ottimizzare gli outcome sanitario ed economico sono:

1) utilizzare alprazolam secondo gli schemi posologici indicati e validati dalla ricerca clinica;

2) adottare tutte le precauzioni necessarie per evitare abusi, iperdosaggi e reazioni avverse.

Utilizzato in modo appropriato, alprazolam può essere considerato una benzodiazepina vantaggiosa per il paziente, per il medico prescrittore, per il SSN e per la società.

\section{BIBLIOGRAFIA}

1. Dawson GW, Jue SG, Brodgen RN. Alprazolam: a review of its pharmacodynamic properties and efficacy in the treatment of anxiety and depression. Drugs 27: 132-147, 1984.

2. Fawcett JA, Kravitz HM. Alprazolam: pharmacokinetics, clinical efficacy, and mechanism of action. Pharmacotherapy 2: 243-254, 1982.

3. Wright CE, Sisson TL, Fleishaker JC, et al. Pharmacokinetics and psychomotor performance of alprazolam: concentration-effect relationship. J Clin Pharmacol 37: 321-329, 1997.

4. Eberts FS, Philopoulos Y, Reinecke LM, et al. Disposition of 14C-alprazolam, a new anxiolytic-antidepressant, in man. Pharmacologist 22: 279, 1980.

5. Kaplan GB, Greenblatt DJ, Ehrenberg BL, et al. Single-dose pharmacokinetics and pharmacodynamics of alprazolam in elderly and young subjects. J Clin Pharmacol 38: 14-21, 1998.

6. Juhl RP, Van Thiel DH, Dittert LW, et al. Alprazolam pharmacokinetics in alcoholic liver disease. J Clin Pharmacol 24: 113-119, 1984.

7. Abernethy DR, Greenblatt DJ, Divoll M, et al. The influence of obesity in the pharmacokinetics of oral alprazolam and triazolam. Clin Pharmacol 9: 177-183, 1984.

8. Greenblatt DJ, Wright CE. Clinical pharmacokinetics of alprazolam: therapeutic implications. Clin Pharmacokin 24: 453-471, 1993.

9. Greenblatt DJ, Harmatz JS, Shader RI. Plasma alprazolam concentrations : relation to efficacy and side effects in the treatment of panic disorder. Arch gen Psychiatry 50: 715-722, 1993.

10. Fabre LF, McLendonDM. A double-blind study comparing the efficacy and safety of alprazolam with imipramine and placebo in primary depression. Curr Ther Res 27: 474-482, 1980.

11. Hobbs WR, Rall TW, Verdoorn TA. Hypnotics and sedatives; ethanol. In “Goodman \& Gilman's The pharmacological basis of therapeutics”, cap 17- IX Edition, McGraw-Hill, New York, 1996.

12. Symposium (autori vari): “GABA and benzodiazepine receptor subtypes: molecular biology, pharmacology, and clinical aspects”, Biggio G \& Costa E Eds., Adv Biochem Psychopharmacol 46: 1-239, 1990.

13. Huybrechts I. The pharmacology of alprazolam: a review. Clin Ther 13: 100-117, 1991. 
14. Van Gool D. Different modes of action of alprazolam in the treatment of panic attacks. Acta Neuropsychiatrica 12 (II): 41-45, 2000.

15. Bonnet MH, Kramer M, Roth T. A dose response study of the hypnotic effectiveness of alprazolam and diazepam in normal subjects. Psychopharmacology 75: 258-261, 1981.

16. Massoud N, Wang RIH. Alprazolam. Drugdex Drug Evaluation, Healthcare series, Micromedex, 2001.

17. Greiss KC, Fogari R. Double-blind clinical assessment of alprazolam, a new benzodiazepine derivative, in the treatment of moderate to severe anxiety. J Clin Pharmacol 20: 693-699, 1980.

18. Kerry RJ, McDermont CM. Alprazolam in the treatment of neurotic anxiety. Pharmacotherapeutica 3: 451-455, 1983.

19. Davison K, Farquharson RG, Khan MC, et al. A double-blind comparison of alprazolam, diazepam, and placebo in the treatment of anxious out-patients. Psychopharmacology 80: 308-310, 1983.

20. Morphy MA. A double-blind comparison of alprazolam and placebo in the treatment of anxious schizophrenic outpatients. Curr Ther Res 40: 551-560, 1986.

21. Tolleston GD, Luxenberg M, Valentine R, et al. An open label trial of alprazolam in comorbid irritable bowel syndrome and generalized anxiety disorder. J Clin Psychiatry 52:502-508, 1991.

22. Itil TM, Polvan N, Egilmez S, et al. Anxiolytic effects of a new triazolobenzodiazepine, U31,889. Curr Ther Res 15: 603-615, 1973.

23. Ballenger JC, Davidson JRT, Lecubrier Y, et al. Consensus statement on panic disorder from the International Consensus Group on Depression and Anxiety. J Clin Psychiatry 59 (Suppl 8): 47-54, 1998.

24. Bennett JA, Moioffer M, Stanton SP, et al. A risk-benefit assessment of pharmacological treatments for panic disorder. Drug Safety 18: 419-430, 1998.

25. Baldwin DS, Birtwistle J. The side effect burden associated with drug treatment of panic disorder. J Clin Psychiatry 59: 39-44, 1998.

26. Rickels K, Schweizer E, Panic disorder: long-term pharmacotherapy and discontinuation. J. Clin Psychopharmacol 18(6 Suppl 2):12S-18S, 1998.

27. Chouinard G, Annable L, Fontaine R, et al. Alprazolam in the treatment of generalized anxiety and panic disorders: a double-blind, placebo-controlled study. Psychopharmacol Bull 19: 115-116, 1983.

28. Gelenberg AJ. Treating panic attacks. Ma Gen Hosp Newsletter Biol Ther Psychiatry 5: 1-2, 1982.

29. Kaplan GB, Greenblatt DJ, Ehrenberg BL, et al. Differences in pharmacodynamics but not pharmacokinetics between subjects with panic disorder and healthy subjects after treatment with a single dose of alprazolam. J Clin Psychopharmacol 20: 338-346, 2000.

30. Nutt DJ, Glue P.Lawson C, et al. Flumazenil provocation of panic attacks : evidence for altered benzodiazepine receptor sensitivity in panic disorder. Arcg Gen Psychiatry 47: 917-925, 1990.

31. Kikka JT, Pitkanen A, Lepola U, et al. Abnormal regional benzodiazepine receptor uptake in the prefrontal cortex in patients with panic disorder. Nucl Med Commun 16: 273-280, 1995.

32. Ballenger JC, Burrows GD, DuPont RL, et al. Alprazolam in panic disorder and agoraphobia: results from a multicenter trial: I. Efficacy in short-term treatment. Arch Gen Psychiatry 45: 413-422, 1988.

33. Schweizer E, Rickels K, Weiss S, et al. Maintenance drug treatment of panic disorder: I. Results of a prospective, placebo-controlled comparison of alprazolam and imipramine. Arch Gen Psychiatry 50: 51-60, 1993.

34. Rickels K, Schweizer E, Weiss S, et al. Maintenance drug treatment of panic disorder: II. Short-and long-term outcome after drug taper. Arch Gen Psychiatry 50: 61-68, 1993.

35. Sheikh JI, Swales PJ. Treatment of panic disorder in older adults: a pilot study comparison of alprazolam, imipramine, and placebo. Int J Psychitary Med 29: 107-117, 1999.

36. Andersch S, Rosenberg NK, Kullingsjo H, et al. Efficacy and safety of alprazolam, imipramine and placebo in treating panic disorder: a Scandinavian multicenter study. Acta Psychiatr Scand 365 (suppl): 18-27, 1991.

37. Mellergard M, Lorentzen K, Bech P, et al. A trend analysis of changes during treatment of panic disorder with alprazolam and imipramine. Acta Psychiatr Scand 365: 28-32, 1991.

38. Davidson JRT. The long-term treatment of panic disorder. J Clin Psychiatry 59: 17-21, 1998.

39. Rosenbaum JF. Treatment-resistant panic disorder. J Clin Psychiatry 58: 61-64, 1997. 
40. Bruce TJ, spiegel DA, Gregg SF, et al. Predictors of alprazolam discontinuation with and without cognitive behavior therapy in panic disorder. Am J Psychiatry 152: 1156-1160, 1995.

41. Bruce TJ, Spiegel DA, Hegel MT. Cognitive-behavioral therapy helps prevent relapse and recurrence of panic disorder following alprazolam discontinuation: a long-term follow-up of the Opeoria and Darmouth studies. $\mathrm{J}$ Consul Clin Psychology 67: 151-156, 1999.

42. Kravitz HM, Fawcett J, Newman AJ. Alprazolam and depression: a review of risks and benefits. J Clin Psychiatry 54(Suppl): 78-84, 1993.

43. Fabre LF. Pilot open label study with alprazolam in outpatients with neurotic depression. Curr Ther Res 19: 661668, 1976.

44. Feighner JP, Aden GC, Fabre LF, et al. Comparison of alprazolam, imipramine and placebo in the treatment of depression. JAMA 249: 3057-3064, 1983.

45. Lenox RH, Shipley JE, Peyser JM, et al. Double-blind comparison of alprazolam versus imipramine in the inpatient treatment of major depressive illness. Psychopharmacol Bull 20: 79-82, 1984.

46. Overall JE, Donache ND, Failace LA. Implications of restrictive diagnosis for compliance to antidepressant drug therapy: alprazolam versus imipramine. J Clin Psychiatry 48: 51-54, 1987.

47. Keller MB, Lavori PW, Goldenberg IM, et al. Influence of depression on the treatment of panic disorder with imipramine, alprazolam and placebo. J Affect Disor 28: 27-38, 1993.

48. Erikson B, Nagy A, Starmark JE, et al. Alprazolam compared to amitriptyline in the treatment of major depression. Acta Psychiat Scand 75: 656-663, 1987.

49. Lapierre YD, Browne M, Oyewumi K, et al. Alprazolam and amitriptyline in the treatment of moderate depression. Int Clin Psychopharmacol 9: 41-45, 1994.

50. Banerji JR, Brantingham P, McEwan GD, et al. A comparison of alprazolam with amitriptyline in the treatment of patients with neurotic or reactive depression. Irish J Med Sci 158: 110-113, 1989.

51. Feighner JP. Open label study of alprazolam in severely depressed inpatients. J Clin Psychiatry 44: 332-334, 1983.

52. Ries RK, Wittkowsky AK. Synergistic action of alprazolam with tranylcypromine in drug-resistant atypical depression with panic attacks. Biol Psychiatry 21:519-526, 1986.

53. Rickels K, Chung HR, Csanalosi IB, et al. Alprazolam, diazepam, imipramine, and placebo in outpatients with major depression. Arch Gen Psychiatry 44: 862-866, 1987.

54. O'Sullivan GH, Noshirvani H, Basoglu M, et al. Safety and side-effects of alprazolam. Controlled study in agoraphobia with panic diosrder. Br J Psychiatry 165: 79-86, 1994.

55. Cassano GB, Toni C, Petracca A, et al. Adverse effects associated with the short-term treatment of panic disorder with imipramine, alprazolam or placebo. Eur Neuropsychopharmacology 4: 47-53, 1994.

56. Gladsjo JA, Rapaport MH, McKinney R, et al. Absence of neuropsychologic deficits in patients receiving long-term treatment with alprazolam-XR for panic disorder. J Clin Psychopharmacol 21: 131-138, 2001.

57. Curran HV, Bond A, O'Sullivan G, et al. Memory functions, alprazolam and exposure therapy: a controlled longitudinal study of agoraphobia with panic diosrder. Psychol Med 24: 969-976, 1994.

58. Kilic C, Curran HW, Noshirvani H, et al. Long-term effects of alprazolam on memory: a 3,5 years follw-up of agoraphobia/panic patients. Psychol Med 29: 225-231, 1999.

59. Griffiths RR, Wolf B. Relative abuse liability of different benzodiazepines in drug abusers. J Clin Psychopharmacol 10: 237-243, 1990.

60. Jurgens SM, Morse RM. Alprazolam dependence in seven patients. Am J Psychiatry 145: 625-627, 1988.

61. Noyes R Jr, Garvey MJ, Cook B, et al. Controlled discontinuation of benzodiazepine treatment for patients with panic disorder. Am J Psychiatry 148: 517-523, 1991.

62. Larson DB, Lyons JS, Hohmann AA, et al. Psychotropics prescribed to the US elderly in the early and mid 1980's: prescribing patterns of primary care practitioners, psychiatrists, and other physiscians. Intern J Ger Psychiatry 6: 63-70, 1991.

63. Ustun T.B., Sartorius N. “Mental illness in general health care”. Ed: Chichester; Willey and sons, 1995.

64. Sartorius N, Ustun TB, Lecubier Y, et al. Depression comorbid with anxiety : results from the WHO study on psychological disorders in primary health care. Br J Psychiatry (Suppl 30): 38-43, 1996. 
65. Berardi D, Berti Ceroni G, Leggieri G, et al. Mental, physical and functional status in primary care attenders. Int J Psychiatry Med 29: 133-148, 1999.

66. Fava M, Rosenbaum JF. Anger attacks in patients with depression. J Clin Psychiatry 60 (suppl 15): 21-24, 1999.

67. Fava M, Rankin MA, Wright EC, et al. Anxiety disorders in major depression. Comprehensive Psychiatry. 41: 97-102, 2000.

68. Angst J, Merikangas KR, Preisig M. Subthreshold syndromes of depression and anxiety in the community. J Clin Psychiatry 58 (Suppl8): 6-10, 1997.

69. Angst J. Comorbidity of mood disorders: a longitudinal prospective study. Br J Psychiatry (Suppl 30): 31-37, 1996.

70. Greenberg PE, Sisitsky T, Kessler RC, et al. The economic burden of anxiety disorders in the 1990s. J Clin Psychiatry 60:427-435, 1999.

71. DuPont RL, Rice D, Miller L, et al. Economic costs of anxiety. Anxiety 2: 167-172, 1996.

72. Greenberg PE, Stiglin LE, Finkelstein SN, et al. Economic burden of depression in 1990. J Clin Psychiatry 54: 405-418, 1993.

73. Simon GE, Ormel G, VonKorff M, et al. Health care costs associated with depressive and anxiety disorders in primary care. Am J Psychiatry 152: 352-357, 1995.

74. Souetre E, Lozet H, Cimarosti I, et al. Cost of anxiety disorders: impact of comorbidity. J Psychosom Res 38 (Suppl 1): 151-160, 1994.

75. Martin P. Impacts médico-socio-économiques des troubles anxieux. L'Encephale 24 : 280-296, 1998.

76. Leon AC, Portera L, Weissman MM. Social costs of anxiety disorders. Br J Psychiatry 166 (Suppl 27): 19-22, 1995.

77. Leon AC, Olfson M, Portera L. Service utilization and expenditures for the treatment of panic disorder. Gen Hosp Psychiatry 19: 82-88, 1997.

78. Salvador-Carulla L, Seguì J, Fernadez-Cano P, et al. Costs and offset effect in panic disorders. Br J Psychiatry 166 (Suppl 27): 23-28, 1995.

79. Kessler RC, McGonagle K, Zhao S, et al. Lifetime and 12.month prevalence of DSM-III-R psychiatric disorders in the United States. Arch Gen Psychiatry 51: 8-19, 1994.

80. Lyons JS, Larson DB, Hromco J. Clinical and economic evaluation of benzodiazepines: a value analysis. Pharmacoeconomics 2: 397-407, 1992.

81. Kahneman D, Twersky A. Choices, values, and frames. Am Psychologist 39: 341-450, 1984.

82. McGlynn EA, Norquist GS, Wells KB, et al. Quality-of-care research in mental health: responding to challenge. Inquiry 25: 157-170, 1988. 